\title{
Removing interference echoes in Philippine radars using a fuzzy logic approach
}

\author{
Pin-Fang $\operatorname{Lin}^{1}$, Lester Kim M. Lagrimas ${ }^{2, *}$, Jung-Bin Wang ${ }^{1}$, Pao-Liang Chang ${ }^{1}$, Wei-Ting Fang ${ }^{1}$, \\ John Grender C. Almario ${ }^{2}$, Ben Jong-Dao Jou ${ }^{3}$, and Esperanza O. Cayanan ${ }^{2}$ \\ ${ }^{1}$ Central Weather Bureau, Taipei City, Taiwan \\ ${ }^{2}$ Philippine Atmospheric, Geophysical and Astronomical Services Administration, Metro Manila, Philippines \\ ${ }^{3}$ Department of Atmospheric Sciences, National Taiwan University, Taipei City, Taiwan
}

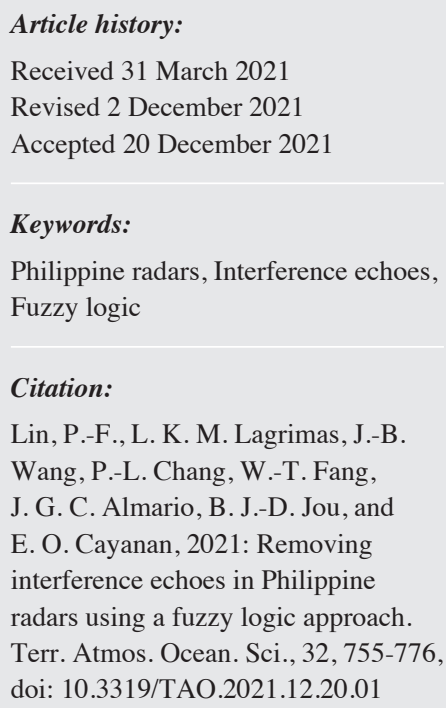

\begin{abstract}
Ideally echoes in radar reflectivity data correspond to precipitating particles, however they do not, and as a result, automated weather radar products that use these data are drastically affected when conditions are not ideal. Weather radar data of the Philippine Atmospheric, Geophysical and Astronomical Services Administration (PAGASA) is one such case that often suffer contamination, in particular by electromagnetic interference and the identification and mitigation of interference echoes is an ongoing problem in radar meteorology in these regions. In order to improve the quality of the data and consequently the automated products, especially for the radar quantitative precipitation estimation (QPE), a fuzzy logic algorithm is applied upon the radar reflectivity data to provide a probability guidance for segregating interference-contaminated echoes from precipitating echoes. Specifically, adequate features to highlight interference characteristics are required for the algorithm to be effective based on prior experiences. This approach is presented in this study to derive membership functions and their relatively objective weights are determined based on the superior result of sensitivity test from interference cases. The result of which produced a value that quantifies the possibility of each bin being affected by interference. Cases that highlighted the interference were examined and demonstrated the ability of the fuzzy logic approach to remove interference echoes from radar reflectivity map. Moreover, the presented method can be feasibly implemented in real-time multi-radar operations as a quality control (QC) aid.
\end{abstract}

\section{INTRODUCTION}

Weather radars are commonly used to estimate rainfall at high spatial and temporal resolution over large regions. However, echoes in radar reflectivity data do not always correspond to precipitating particles. Instead (common non-weather) echoes on radar may be due to biological targets such as insects, birds or wind-borne particles, due to anomalous propagation (AP; sometime over the sea, called sea clutter) or ground clutter (GC) or due to test and interference patterns that inadvertently seep into the consequent products. Quality control (QC) of radar data is one of the important issues related to improving radar quanti-

\footnotetext{
* Corresponding author

E-mail:kim.lagrimas@meteopilipinas.gov.ph
}

tative precipitation estimate (QPE) from radar measurements, especially the challenge still exists to derive accurate radar quantitative precipitation forecast $(\mathrm{QPF})$ on weather systems. Radar echoes caused by non-meteorological targets may introduce significant biases in precipitation fields, resulting in an abrupt rainfall accumulation gradient and spatial discontinuity, even rainfall nowcast. Errors in radar rainfall estimates have been shown to propagate nonlinearly in hydrologic forecasts of streamflow (e.g., Droegemeier et al. 2000; Ogden et al. 2000).

This study is supported and executed under the Project VOTE (Volcanos, Ocean, Typhoon, and Earthquake) which was the collaboration between CWB (Central Weather Bureau, Taiwan) and PAGASA (Philippine Atmospheric, 
Geophysical and Astronomical Services Administration) through the TECO-MOST/MECO-DOST (Taipei Economic and Cultural Office-Ministry of Science and Technology/ Manila Economic and Cultural Office-Department of Science and Technology). The primary purpose of this study is to develop a flexible approach based on fuzzy logic concepts to segregate interference-contaminated echoes from precipitating echoes for the Philippine radar network, since the reflectivity map was often suffered contamination by electromagnetic interference. The PAGASA currently operates 17 radars nationwide to survey weather events with locations as shown in Fig. 1. As such, they provide a critically important dataset to those living within their coverage area, such as during the tropical cyclone season and for the Radar Rainfall Warning System of PAGASA, when significant property damages and loss of human lives can occur. Unfortunately, interference echoes in the radar network are emerging as a serious data issue, as observed in the Philippine radar network (Fig. 2). Saltikoff et al. (2016) indicated that the threat of wireless technology to a weather radar is observed as a presence of an echo line oriented radially toward the radar and radar data, the source of being radiation from nearby telecommunication links. Or radio frequency interference can prevent the retrieval of meteorological information by a weather radar in affected azimuthal sectors and present false data that might be mistaken for actual atmospheric observations. Accordingly, a completely automated algorithm that can remove non-weather echoes, such as the interference from wireless technology (Lakshmanan et al. 2007) or a new radio frequency interference (RFI) filter algorithm for weather radars proposed in the two-dimensional (2D) range-time/sample-time domain (Cho 2017), would be very serviceable in decreasing contaminations in the radar reflectivity moment and in improving the performance of automated weather radar processes.

Numerous researches have been devoted to improving radar data quality. For instance, intensity of ground clutter and of echoes of anomalous propagation and its elimination (Lee et al. 1995); AP Clutter Mitigation Scheme (Kessinger et al. 2001, 2002; Ellis et al. 2003) to improve the WSR-88D radar-derived rainfall estimates by enhancing the quality of the data through the identification and removal of certain contaminants; use of three-dimensional reflectivity structure for automated detection and removal of AP echoes in radar data (Steiner and Smith 2002); identification and removal of non-precipitation echoes using the characteristics of radar echoes (Lee et al. 2005; Cho et al. 2006); uses statistics to highlight clutter characteristics that quantifies the possibility of each bin being affected by clutter to remove ground clutter and sea clutter (Berenguer et al. 2006), and so on. Many different approaches have been proposed to detect, mitigate, and eliminate non-precipitating echoes or improve radar data quality by using radar observations, such as threshold-based, polarimetric observations, deci- sion trees, fuzzy logic, neural networks, or combinations of these therein. However, fuzzy logic algorithms have been widely used for the mitigation of non-meteorological echo due to their simplicity. For instance, the Radar Echo Classifier (REC) based on fuzzy logic was deployed on the National Weather Service's Weather Surveillance Radar-1988 Doppler (WSR-88D) (Kessinger et al. 2003); fuzzy logic algorithm also is used to identify and filter clutter echoes based on the characteristics of radar echoes (Berenguer et al. 2006; Cho et al. 2006); identification and removal of non-meteorological echoes in dual-polarization radar data based on a fuzzy logic algorithm (Gourley et al. 2007; Ye et al. 2015; Kilambi et al. 2018; Overeem et al. 2020); etc. Through the mentioned studies, it has been proven that fuzzy logic method possesses the ability to separate weather and non-weather patches in reflectivity map to improve the radar data quality. Nevertheless, it is noteworthy that none of the reported approaches intently constructed in the case of interference echoes embedded in precipitation echoes, a problem that remains a challenge today for radar data quality control. Taking strengths and weaknesses of past approaches into consideration, a fuzzy logic QC procedure for interference identification and removal has been developed in this study that makes use of the one and two dimensional reflectivity structure.

An adequate tool that straightforwardly integrates observational factors is essential as well as can quantitatively provide the interference information in real-time operations. For example, the capability of individual factors such as to identify the appearance of interference can improve the radar data quality. The primary purpose of this study is to develop a flexible algorithm based on fuzzy logic concepts, to provide interference-echo detection based on radar echo characteristics, and automate quality control focusing on removing interference echoes. This paper is organized as follows: In section 2, the radar data used and the methodology of the fuzzy logic algorithm to identify interference echoes are described. The performance of interference echo removal from reflectivity field and the subsequent application, radar QPE, are presented in section 3, which are followed by a summary, conclusions, and suggestions for future work in section 4 .

\section{DATA AND METHODOLOGY}

Of the 17 radars that make up the PAGASA network (Fig. 1), the Hinatuan (HINA), Mactan (MACT), and Tagaytay (TAGA) radars each regularly observe interference signals that result in the contamination of their weather radar products. In this study, the contaminated HINA radar data is used characterize the interference signals and, from that, construct the algorithms for removing these interference echoes. The membership functions for the fuzzy logic are derived from either observed or derived radar parameters, 


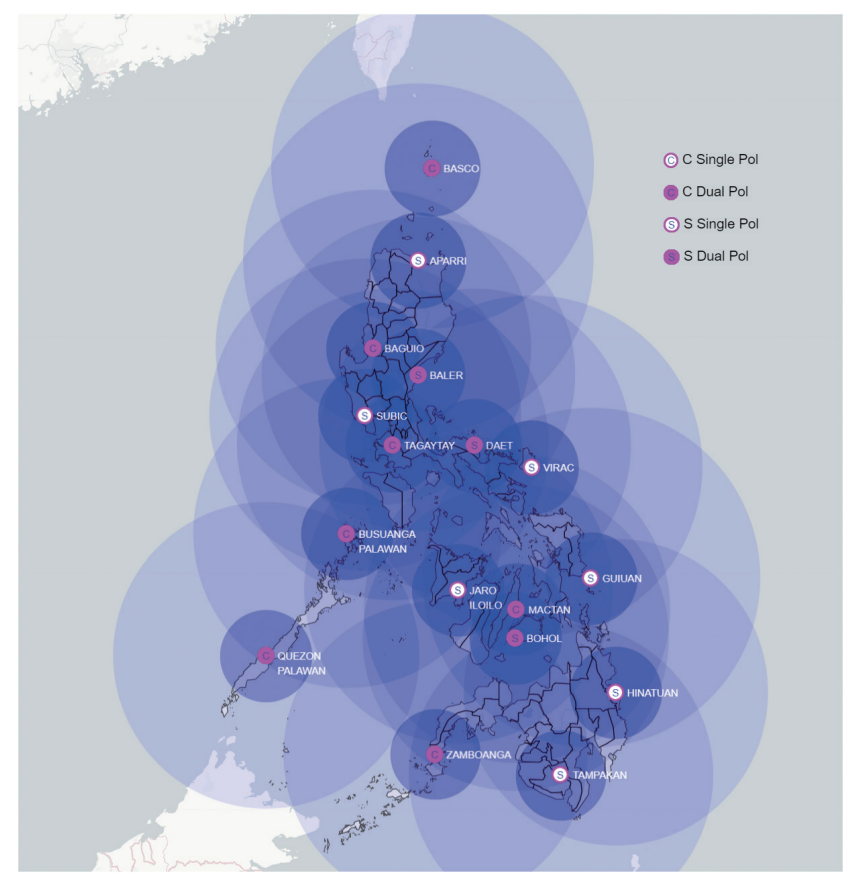

Fig. 1. The weather radar network in the Philippines. Range rings of light purple and deep purple represent $480 \mathrm{~km}$ of surveillance mode and 120 $\mathrm{km}$ of Doppler mode centered at each radar site, respectively. Radar sites are labeled with the microwave band and un-highlighted and highlighted circles respectively symbolize single and dual polarization.

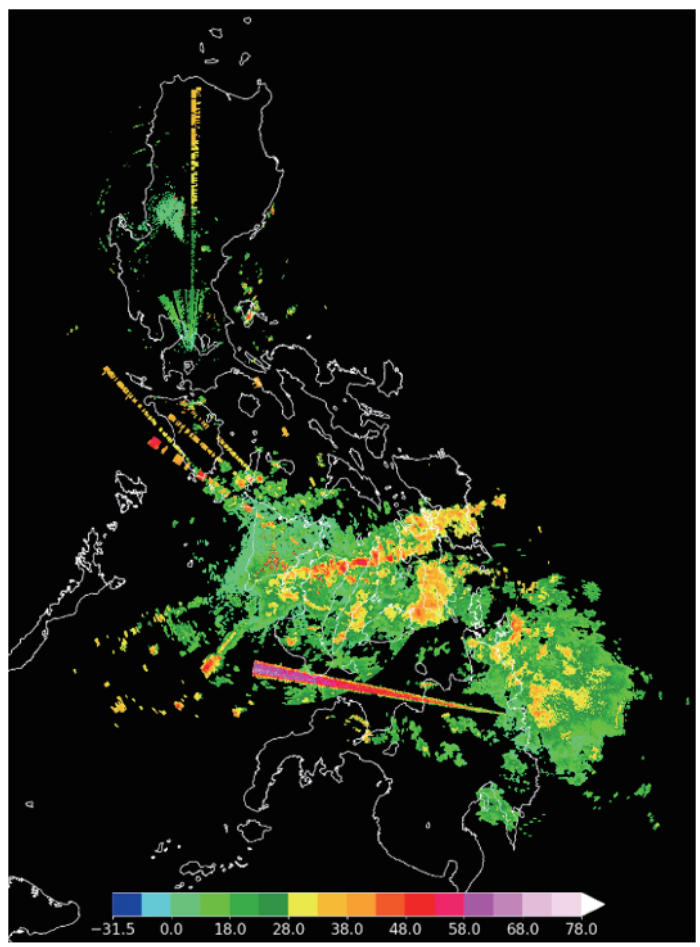

Fig. 2. The composite radar reflectivity map from the Philippine radar network at 1630 UTC 10 November 2018. 
and the weights for these membership functions are determined based on maximizing the skill score CSI (Critical Success Index, Donaldson et al. 1975). Once the algorithm was constructed, the MACT and TAGA radar data are used to evaluate its performance.

\subsection{Radar Data}

The first weather surveillance radar of PAGASA in the Philippines was installed in Manila in 1963, and in 1971, five new radar stations were added and all were linked with the Manila radar station to form the country's initial weather radar surveillance network. Currently, there are seventeen operational radars of PAGASA in the Philippines, as shown in Fig. 1. With the radar network, weather systems can be monitored even before it arrives in the Philippines. The Modified VCP (Volume Coverage Pattern) 11 was chosen by the Technical Working Group (TWG) as the primary scanning strategy for PAGASA radars because of its better high and low level coverage pattern, which is better for tropical weather condition according to NOAA (available online at https://www.roc.noaa.gov/WSR88D/PublicDocs/ NewTechnology/New VCP Paradigm Public Oct 2015. pdf). The horizontal range of the Modified VCP 11 Scanning Strategy Doppler mode was set to $120 \mathrm{~km}$ as a compromise between the maximum Nyquist velocity and coverage range. In addition to the coverage pattern, the lowest two-elevation scans of 0.5 degree and 1.5 degree having low pulse repetition frequency (PRF) and long pulse width were added to the scanning strategy in order to provide longer ranges up to $480 \mathrm{~km}$.

Radar data utilized in this study for developing the approach to remove the non-precipitation echoes, interferences, is primary from the HINA radar of PAGASA. Technical specifications of the HINA radar are summarized in Table 1. Other radar data, such as the MACT and TAGA radars, which has high occurrence of interferences are also used to validate the improvement of data quality using developed fuzzy logic QC algorithm. In this study, a total of 1000 observational radar $0.0^{\circ}$ PPI (Plan Position Indicator) files (listed in Table 2) from the HINA radar are collected as a dataset, including 500 files of distinct interference patterns without obvious precipitation events and the other 500 files of only precipitation patches. In particular, the algorithm was developed and executed in polar coordinates so that the data recording remains in the same format. While Interpolation of the data could have been converted to a Cartesian grid which could have simplified data management and processing, undesired range-dependent artifacts would have been introduced (e.g., Trapp and Doswell III 2000). They are meticulously analyzed to produce 6 feature curves as described in section 2.2.

\subsection{Features for Interference Removal}

The fuzzy logic algorithm developed in this study is to remove interference observed from the HINA radar. The features decided to be used in the fuzzy logic approach are dominantly referred from Steiner and Smith (2002), Kessinger et al. (2003), Berenguer et al. (2006), and Gourley et

Table 1. Technical specifications of the HINA, MACT, and TAGA radars in Philippines.

\begin{tabular}{|c|c|c|c|}
\hline Radar & HINA & MACT & TAGA \\
\hline Coordinates & $\begin{array}{c}8^{\circ} 22^{\prime} 02.37 " \mathrm{~N} \\
126^{\circ} 20^{\prime} 18.73^{\prime \prime} \mathrm{E}\end{array}$ & $\begin{array}{l}10^{\circ} 19^{\prime} 21.80^{\prime \prime} \mathrm{N} \\
123^{\circ} 58^{\prime} 49.1^{\prime \prime} \mathrm{E}\end{array}$ & $\begin{array}{l}14^{\circ} 08^{\prime} 31.7^{\prime \prime} \mathrm{N} \\
121^{\circ} 01^{\prime} 20.2^{\prime \prime} \mathrm{E}\end{array}$ \\
\hline Manufacturer/Model & EEC/DWSR-8501S & EEC/DWSR-2501C & EEC/DWSR-2501C \\
\hline Bandwidth/Polarization & S-band/Single polarization & C-band/Dual polarization & C-band/Dual polarization \\
\hline Peak power/Frequency & $850 \mathrm{~kW} / 2705 \mathrm{MHz}$ & $250 \mathrm{~kW} / 5610 \mathrm{MHz}$ & $250 \mathrm{~kW} / 5610 \mathrm{MHz}$ \\
\hline Pulse width (Surveillance/Doppler Mode) & $2 / 0.8 \mathrm{~ms}$ & $2 / 0.8 \mathrm{~ms}$ & $2 / 0.8 \mathrm{~ms}$ \\
\hline Antenna Speed & $12 \%$ sec & $12 \%$ sec & $12 \%$ sec \\
\hline Elevation & $37 \mathrm{~m}$ & $46 \mathrm{~m}$ & $782 \mathrm{~m}$ \\
\hline Beam width/Range resolution & $1 \% 500 \mathrm{~m}$ & $1 \% / 500 \mathrm{~m}$ & $1 \% / 500 \mathrm{~m}$ \\
\hline \multirow{4}{*}{$\begin{array}{c}\text { Number of angles/Elevation angles } \\
\text { (degrees) }\end{array}$} & \multicolumn{3}{|c|}{ Surveillance Mode } \\
\hline & $2 / 0,1.5$ & $2 / 0.5,1.5$ & $2 / 0.5,1.0$ \\
\hline & \multicolumn{3}{|c|}{ Doppler Mode } \\
\hline & $\begin{array}{c}14 / 1.0,2.0,2.4,3.4,4.3,5.3,6.2 \\
7.8,8.7,10.0,12.0,14.0,16.7 \\
19.5\end{array}$ & $\begin{array}{c}14 / 0.5,1.52 .4,3.4,4.3,5.3,6.2 \\
7.8,8.7,10.0,12.0,14.0,16.7 \\
19.5\end{array}$ & $\begin{array}{c}14 / 0.5,1.5,2.4,3.4,4.3,5.3,6.2 \\
7.8,8.7,10.0,12.0,14.0,16.7 \\
19.5\end{array}$ \\
\hline $\begin{array}{c}\text { Maximum range (Surveillance/Doppler } \\
\text { Mode) }\end{array}$ & $480 / 120 \mathrm{~km}$ & $480 / 120 \mathrm{~km}$ & $480 / 120 \mathrm{~km}$ \\
\hline Volume cycle interval & 10 minutes & 10 minutes & 10 minutes \\
\hline
\end{tabular}


Table 2. The files observed from the HINA radar of PAGASA used in deriving the feature curves.

\begin{tabular}{|c|c|c|c|c|c|}
\hline \multicolumn{3}{|c|}{ Interference } & \multicolumn{3}{|c|}{ Weather } \\
\hline Year/Month & day & Number of files & Year/Month & date & Number of files \\
\hline \multirow[t]{7}{*}{2017.10} & 06 & 22 & 2017.10 & 02 & 5 \\
\hline & 07 & 36 & & 29 & 7 \\
\hline & 08 & 3 & & 30 & 11 \\
\hline & 09 & 14 & 2017.11 & 07 & 11 \\
\hline & 10 & 33 & & 08 & 5 \\
\hline & 11 & 38 & & 10 & 12 \\
\hline & 12 & 32 & & 11 & 22 \\
\hline \multirow[t]{11}{*}{2017.11} & 01 & 3 & & 12 & 1 \\
\hline & 02 & 2 & & 14 & 12 \\
\hline & 03 & 1 & & 15 & 28 \\
\hline & 08 & 3 & & 16 & 21 \\
\hline & 09 & 3 & & 28 & 15 \\
\hline & 12 & 4 & & 30 & 9 \\
\hline & 14 & 3 & 2017.12 & 02 & 8 \\
\hline & 15 & 33 & & 05 & 8 \\
\hline & 28 & 2 & & 11 & 12 \\
\hline & 29 & 4 & & 12 & 10 \\
\hline & 30 & 7 & & 13 & 18 \\
\hline \multirow[t]{24}{*}{2017.12} & 01 & 17 & & 14 & 21 \\
\hline & 02 & 13 & & 15 & 9 \\
\hline & 03 & 16 & & 19 & 6 \\
\hline & 04 & 13 & & 20 & 44 \\
\hline & 05 & 5 & & 21 & 82 \\
\hline & 06 & 16 & & 22 & 34 \\
\hline & 07 & 13 & & 23 & 17 \\
\hline & 08 & 11 & & 25 & 26 \\
\hline & 11 & 1 & & 27 & 41 \\
\hline & 12 & 2 & & 31 & 5 \\
\hline & 16 & 13 & & & \\
\hline & 17 & 23 & & & \\
\hline & 18 & 7 & & & \\
\hline & 19 & 9 & & & \\
\hline & 20 & 3 & & & \\
\hline & 22 & 2 & & & \\
\hline & 24 & 31 & & & \\
\hline & 25 & 21 & & & \\
\hline & 26 & 8 & & & \\
\hline & 27 & 1 & & & \\
\hline & 28 & 1 & & & \\
\hline & 29 & 16 & & & \\
\hline & 30 & 15 & & & \\
\hline & Total & 500 & & Total & 500 \\
\hline
\end{tabular}


al. (2007), and their relatively proper weights are determined based on the superior result of sensitivity test (not shown) (Berenguer et al. 2006) from 500 obvious interference files. For the specific characteristics of interference echoes, the basic radial velocity (VEL) moment was the first feature utilized in the study since many (Kessinger et al. 2003; Berenguer et al. 2006; Gourley et al. 2007) can successfully be used to characterize non-precipitation echoes. The other 5 features derived from the base data fields also used in this study to represent a subjective choice of the characteristics which are expected would distinguish interference and weather echoes. The purpose of the 5 features is to focus on extracting interference characteristics (Saltikoff et al. 2016): (1) When the radar receiver receives an interfering signal from a continuously radiating source, such as from telecommunication links, the interference observed as lines that generally oriented radially in the radar image; (2) If the interfering transmitter is near the radar, the disturbance will be observed as a sector that can be as much as several tens of degrees wide; (3) Some of these echoes have reflectivities that are stronger than the strongest typical weather-related echoes, and can have rapid changes radially. Therefore, the algorithms rely on the shape of the echoes which are a few rays wide, and the texture of neighborhood helps separate interferences from weather echoes. The overall procedure of developing the fuzzy logic approach for identifying interference is illustrated in Fig. 3, which will be stated in section 2.3. The 6 features are the following.

Features Coverage Ratio in Radial (CR) and Azimuthal Ratio (AZR) are to calculate the percentage of valid gates in radial and azimuthal directions (Kessinger et al. 2003), respectively. In a real weather echo map, it's infrequent that the high percentage of covering by a weather system in a whole beam. Feature $\mathrm{CR}$ is calculated as follows:
$C R_{\left(y_{a, b}\right)}=\frac{N_{\text {valid gates }}}{N_{\text {total gates }}} \times 100 \%$

where $a$ and $b$ are the ordinal number of gate and beam, respectively; $y_{(a, b)}$ is the echo value at $a$ th gate and $b$ th beam; $N_{\text {valid gates }}$ is the number of echo values greater than $0 \mathrm{dBZ}$ and $N_{\text {total gates }}$ is the total number of gates in a ray. If considering the $N$ is the total number of gates in azimuthal direction, the $C R_{\left(y_{a b}\right)}$ will be $A Z R_{\left(y_{a, b}\right)}$.

While Feature Texture of Reflectivity (RTEX) (Kessinger et al. 2003; Berenguer et al. 2006; Gourley et al. 2007) is the sum of the differences between the data bin and the adjacent data bins within the analysis box, also called spatial variability. Feature RTEX is expressed as a root-meansquare difference of the input field reflectivity within a 3 rays by 3 gates pix window to take into consideration for both of radial and azimuthal directions. This pixel window increases with range; thus, the texture variable will have a range dependence. Feature RTEX can also indicate the chap structure in an interference rays. Feature RTEX is defined as the following:

$R T E X_{\left(y_{a, b}\right)}=\sqrt{\frac{\sum_{i=-(m-1) / 2}^{i=(m-1) / 2} \sum_{j=-(n-1) / 2}^{j=(n-1) / 2}\left(y_{a, b}-y_{a+i, b+j}\right)^{2}}{m \times n}}$

where $a$ and $b$ are the ordinal number of gate and beam, respectively; $y_{(a, b)}$ is the echo value at $a$ th gate and $b$ th beam; $i$ is the index along radial and $j$ is the index along azimuth; $m$ and $n$ are set to be 3 .

The feature Spin Change (SC), which was proposed by Smith et al. (1996) and has been used by Grecu and Krajewski (2000), Steiner and Smith (2002), Kessinger et al. (2003), and Berenguer et al. (2006). Feature SC indicates

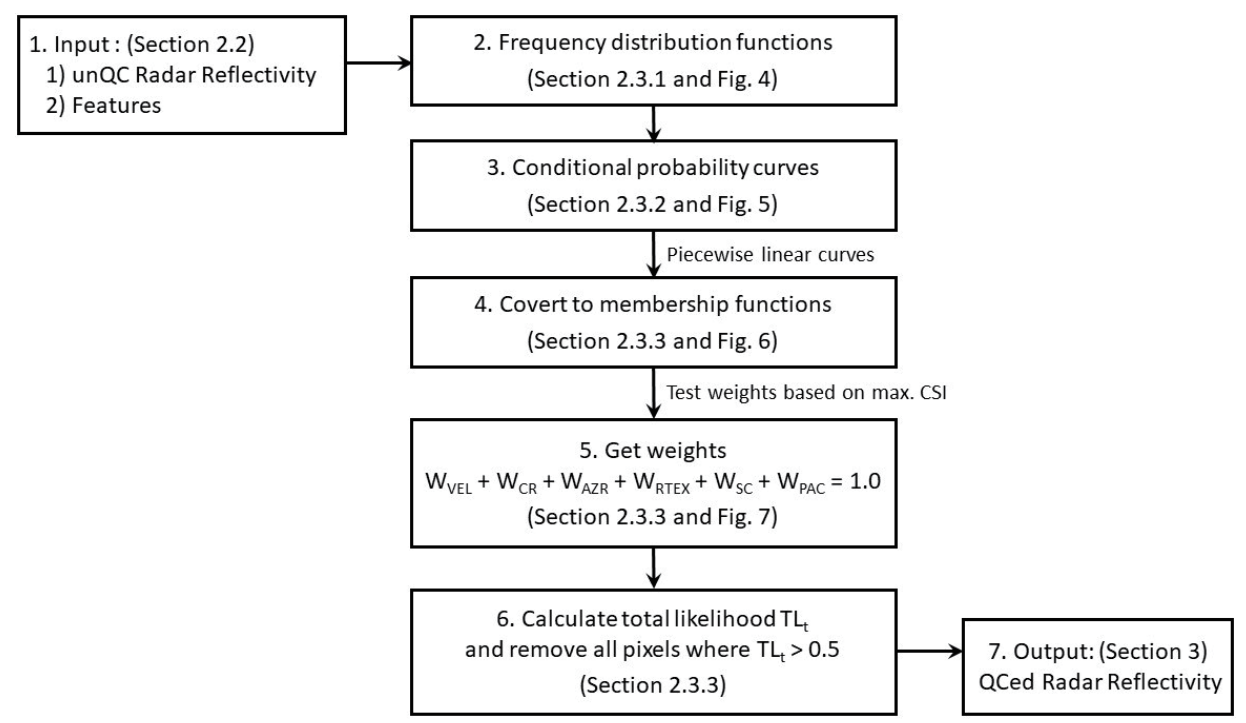

Fig. 3. Schematic diagram of the fuzzy logic algorithm for identifying interference echoes. 
the number of reflectivity fluctuations larger than $2 \mathrm{dBZ}$ within an in a 5rays by 5 gates pixel window, expressed as a percentage of all possible "spin" changes. A reflectivity increase (decrease) in radial direction from one pixel to the next by more than $2 \mathrm{dBZ}$ would cause the spin to point up (down), whereas reflectivity fluctuations smaller than $2 \mathrm{dBZ}$ are deemed insignificant and thus have no effect on the spin setting. Feature SC can be regarded as a data roughness.

$S C_{\left(y_{a, b}\right)}=\frac{\sum_{i=-m}^{m} \sum_{j=-n}^{n} g\left[y_{(a, b)}, y_{(a+i, b+j-1)}\right]}{(2 m+1) \times(2 n+1)} \times 100 \%$

$g\left[y_{(a, b)}, y_{(a+i, b+j-1)}\right]$

$= \begin{cases}1, & \text { if } a b s\left[y_{(a, b)}-y_{(a+i, b+j-1)}\right]>2 d B Z \\ 0, & \text { else }\end{cases}$

where $m$ and $n$ are set to be 2, and other parameters are the same definitions as above.

Feature Pixel-based Azimuth Continuity (PAC) (Steiner and Smith 2002; Berenguer et al. 2006; Gourley et al. 2007) focuses on calculating the percentage of isolated pixels. It aims to detect interference rays by getting the percentage of continuity and smoothness of neighbor gates less than or equal to $15 \mathrm{dBZ}$ in azimuthal direction. The expression of PAC is:

$P A C_{\left(y_{a, b}\right)}=\frac{\sum_{i=-(m-1) / 2}^{i=(m-1) / 2}\left\{1_{\left.\left(i f\left|y_{a, b+1}-y_{a, b}\right| \leq 15\right)\right\}}\right\}}{m-1} \times 100 \%$

where $m$ is equal to 11 and other parameters are the same definitions as above.

Many studies based on fuzzy logic algorithms showed dual-polarization radar parameters, such as differential reflectivity $\left(Z_{D R}\right)$, cross-correlation $\left(\rho_{H V}\right)$, and specific differential phase $\left(K_{D P}\right)$, can be utilized for removing interference echoes embedded in meteorological echoes (Gourley et al. 2007; Ye et al. 2015; Kilambi et al. 2018; Radhakrishna et al. 2019; Overeem et al. 2020). However, the PAGASA network is not fully dual-pol (Fig. 1), only the features derived from single-pol radar parameters are employed in this study.

\subsection{Fuzzy Logic}

The overall procedure of developing the fuzzy logic approach for segregating interference-contaminated echoes from precipitating echoes is now presented; the procedure is also illustrated in Fig. 3. The more detailed explanations on each step will be stated in the following sections. The steps in Fig. 3 are:

(1) Frequency distribution functions (Step 2) - For each of the 6 features used (Step 1) determine its frequency of feature values separately for the interference and precipitation echoes (see section 2.3.1 and Fig. 4).

(2) Conditional probability curves (Step 3 ) - For the interference echoes, convert the above frequency distribution functions to conditional probability curves with values between 0 and 1 (see section 2.3.2 and Fig. 5). Simultaneously, subtract the probability of interference echoes from 1 to obtain the probability for the precipitation echoes.

(3) Membership functions (Step 4) - convert the conditional probability curves for each feature to membership functions (section 2.3.3 and Fig. 6), via a simple piecewise linear transformation.

(4) Feature weight (Step 5) - Determine weights for each of the feature membership functions based on the weights that give the maximum skill score (CSI) for discriminating interference echoes from weather echoes (see section 2.3.3 and Fig. 7).

(5) Fuzzy QC (Step 6) - From Step 5 use the weights to obtain a total likelihood value for interferences. For this purpose, a resulted total likelihood of $>0.5$ is considered a yes for removing interferences (see section 3 ).

\subsubsection{Frequency Distribution Functions}

The following is to identify the feature curves from the dataset. For each feature $F_{k}$, conditional to echo type $t$ (weather or interference echoes) can be expressed as follow (Berenguer et al. 2006),

$$
\begin{aligned}
F_{k, t}(x) & =p\left(F_{k}=x \mid \text { echo type }=t\right) \\
& =\frac{n\left(F_{k}=x \cap \text { echo type }=t\right)}{n(\text { echo type }=t)}
\end{aligned}
$$

where $F_{k, t}(x)$ is frequency, subscript $k$ indicates the different features, $n\left(F_{k}=x \cap\right.$ echo type $\left.=t\right)$ stands for the number of data bins where $F_{k}=x$ and the echo has been classified as type $t ; n($ echo type $=t)$ is the total number of echo classified as echo type $t ; p$ is the probability to be the echo type $t$ at a data bin with a $x$ value.

Figure 4 presents the feature curves (also called frequency distribution functions) for each feature by a total of $10000.0^{\circ} \mathrm{PPI}$ files (where equal interference and precipitation files are used) from the HINA radar. Each of these features were selected because they exhibit an obvious potential to be used for separating precipitation and interference echoes, however they are not fully successful when used separately. For example, the feature curve for radial velocity (Fig. 4a) shows that the interference signal can be clearly identified at $0 \mathrm{~m} \mathrm{~s}^{-1}$, but, in the same region, precipitation echoes also exists. This means radial velocity, with some other features, will have an ability to distinguish interference echoes from precipitation echoes. Similarly, the curves 
of the other 5 features (Figs. $4 \mathrm{~b}$ - f) also exhibit features that imply good separation between interference and precipitation. None of these features by themselves can discriminate between precipitating and non-precipitating areas. Feature curves of RTEX, SC, and PAC come with two higher frequency peaks at different value range from interference and precipitation dataset. It is believed that the more dataset for interference cases is collected, the smoother the curve tendency will become.

\subsubsection{Conditional Probability Curves}

After the normalized frequency distributions for each feature $F_{k}$, convert the above feature curves to conditional probability curves with value between 0 and 1 . The conditional probability curve of a data bin is affected by a certain echo type $t$ when $F_{k}=x$, the expression is (Berenguer et al. 2006):

$$
\begin{aligned}
p_{k, t}(x) & =p\left(\text { echo type }=t \mid X_{k}=x\right) \\
& =\frac{n\left(X_{k}=x \cap \text { echo type }=t\right)}{n\left(X_{k}=x\right)}
\end{aligned}
$$

where $p_{k, t}(x)$ is probability, $n\left(X_{k}=x\right)$ is the total number of echo type $t$ when $X_{k}=x$. The conditional probability curves could be calculated with different value $x$. In this study, $p_{k, t}(x)$ indicates the conditional probability curve for interference echo. $1-p_{k, t}(x)$ exhibits the conditional probability curve for precipitation echo. If $p_{k, t}(x)$ or $1-p_{k, t}(x)$ is close to 0.5 , it means this feature have no the capability of recognizing an echo is interference or weather echo.

Figure 5 presents the conditional probability curves for each feature from the feature curves as shown in Fig. 4. Similarly, the conditional probability curve of the observation field VEL (Fig. 5a) shows it exhibits different occurrence probabilities between weather and interference echoes. For the conditional probability curves of the other 5 features (Figs. 5b - f), display the remarkable peak value window of interference to distinguish interference echoes from weather echoes. The conditionally probability curves for all 6 features indicate interference signals similar to those of the feature curves. As stated above, feature VEL of observation moments, and the derived features CR, AZR RTEX, SC, and PAC can be adequately selected as the features for the identification and removal of interference echoes from the HINA radar by using fuzzy logic algorithm.

These 6 conditional probability curves are utilized to develop an algorithm to discriminate the interference characteristics from radar data, with the favorable interference conditions for each feature defined as when the probability is above 0.5 . These probability values are then used to determine potential candidates and these selected candidates are then combined, with the object of reducing the overall un- certainties and improving discrimination success, in an algorithm based on fuzzy logic concepts (Berenguer et al. 2006).

\subsubsection{Membership Functions and Weightings}

In general, radar data $\mathrm{QC}$ procedures in this study are that radar data pixels are processed to classify weather and interference signals with either exact true or false. However, fuzzy logic assigns continuous values in between 0 and 1 as likelihood that represents the definable degree of a phenomenon by combining features which probably exhibit its characteristics. Membership functions of fuzzy logic are used to convert feature values in order to get the likelihood and sum up these likelihoods with respective weights.

In this study, the feature values were defined through artificially classified radar dataset as interference radar data, and followed by deriving the conditional probability and membership functions. Best weights for each membership functions were determined by optimization of CSI record, that will be described as follow.

The fuzzy logic approach is composed of three basic processes: (1) Determination of membership functions for each conditional probability curve (Cornman et al. 1998). The membership function is a simple piecewise linear functions based on the conditional probability curves from collected data. (2) Determination of the weights to be assigned to each membership function (e.g., Heske and Heske 1996; Liu and Chandrasekar 2000; Berenguer et al. 2006; Lin et al. 2012). (3) Producing a final likelihood value by summing of up these likelihoods with and their respective weights.

The shape of membership functions should be similar to the shape of conditional probability curves. Because there is some degree of subjectivity involved in producing membership functions, they are usually defined as simple piecewise linear curves (e.g., Lakshmanan 2000; Berenguer et al. 2006; Lin et al. 2012). Membership functions perform the conversion of measurement data into scaled, unitless numbers that indicate the correspondence or membership level of the data to the interference echoes. Values of membership functions are converted into a likelihood $L_{k, t}\left(x_{k}\right)$ between 0 (lowest grade of membership) and 1 (highest grade of membership). Figure 6 presents these membership functions subjectively determined by linearizing in a piecewise manner the conditional probability curve of Fig. 5.

After establishing the membership functions, the next step is to assign weights to each membership function. In this step, the values from individual membership functions for a given file are weighted and summed to produce a total likelihood value $\left(T L_{t}\right)$ for a data bin. This is expressed by the equation below,

$T L_{t}=\frac{\sum L_{k, t}\left(x_{k}\right) \times W_{k, t}}{\sum W_{k, t}}$ 
where $L_{k, t}\left(x_{k}\right)$ is the likelihood of membership function for each feature $x_{k}$, and its $W_{k, t}$ is the membership function weight for interference echoes.

In other words, the determination is obtained by converting the features to dimensionless likelihood values using the membership functions shown in Fig. 6, weighting the importance of each likelihood value and summing. The total likelihood value of interference varies from 0 to 1 . In this study, a total likelihood value $>0.5$ is treated as a threshold for interference-contaminated data bin, and this data bin will be removed from a radar map.

The weights selected for each membership function were based on assigning weights between 5 to $30 \%$, increasing every $5 \%$, to each membership function until a maximum CSI score for the set of features was obtained. In this study, 4221 weight combination for the 6 features are gained. The assigned weights for each 6 features for HINA radar is shown in Fig. 7.

The CSI, also known as the threat score (TS, Bermowitz and Zurndorfer 1979), has been used in many method assessments. The other two skill scores, the probability of detection (POD) and the false alarm rate (FAR), were also calculated as follows (Panofsky and Brier 1965):

$$
\begin{aligned}
& C S I=\frac{h}{(h+m+f)} \\
& P O D=\frac{h}{(h+m)} \\
& F A R=\frac{f}{(h+f)}
\end{aligned}
$$

where $h, m$, and $f$ are defined as hits, misses, and false alarms, respectively. The hits represents correctly identified interference echoes, misses represents incorrectly identified interference echoes, and false alarms are weather echoes incorrectly considered as interference echoes.

In this study, hits, misses, and false alarms counted by files, not data bins. In this study, each data bin in each file was flagged as interference or precipitation echo artificially $\left(\right.$ flag $\left._{\text {artfc }}\right)$, and also flagged by fuzzy logic QC approach $\left(\right.$ flag $\left._{f u z z y}\right)$. A hit, miss, or false alarm for each file dominated the residue of data bin flags after fuzzy logic QC algorithms. These flags were then counted before (NunQC by flag $\left._{a r t f c}\right)$ and after $\left(N Q C e d\right.$ by flag $\left.g_{f u z z y}\right)$. To limit, the number of the total data bins only those with reflectivity $>0$ dBZ were counted. The resulted NQCed was then divided by $N u n Q C$ to obtain the error percentage. For an interference file, if the percentage of data bins kept (flag $g_{\text {artf } c}$ is interference, but flag $_{\text {fuzzy }}$ is precipitation) was less than or equal to Error $_{\text {threshold }} \%$, then this file will be considered a hit. In contrast, the constraint is $N Q C e d / N u n Q C$ should be greater than or equal to (1-Error threshold $)$ to be considered a hit for a weather file. This methodology is described in detail in Table 3. From this experience, using higher error threshold, result in less efficacy of the fuzzy logic QC in removing interference echoes. In this study, the error threshold $10 \%$ in Table 3 was chosen to assess the fuzzy logic as the results obtained using this threshold contain a high efficacy factor.

\section{EVALUATIONS}

In the strictest sense, an ideal fuzzy logic QCed result means while interferences overlapped with precipitation reflectivities are removed, and precipitation reflectivities are still kept. In this section, five representative cases are presented that illustrates the performance of the fuzzy logic algorithm to remove interference echoes with success from radar reflectivity map data. Moreover, some characteristic examples that demonstrate the finer features of the fuzzy logic algorithms are individually analyzed. There are two observed datasets used in the evaluation. One is the calibration dataset, which are observations made by the HINA radar of PAGASA that is used to develop the fuzzy logic algorithm. Besides the HINA radar, interference echoes are also frequently observed by Tagaytay (TAGA) and Mactan (MACT) radars of PAGASA (the technical specifications of the TAGA and MACT radars are summarized in Table 1). Consequently, the other dataset from these two radar is the validation dataset, and is used for the performance evaluation of the fuzzy logic algorithm on the interference removal. These will be shown in the following sub-sections. Afterward, each of the 6 membership function likelihoods and the results obtained for each case will be addressed, and followed by their effects on radar QPE from $u n Q C$ and $Q C e d$ reflectivity.

\subsection{Calibration Dataset}

The first case to be examined is an interference case of the calibration dataset that was observed at 0130 UTC 01 November 2017 from the HINA radar, shown in Fig. 8. There are only line-pattern interference echoes in the radar reflectivity map under a clear weather pattern. After weighting and summing to obtain the final likelihood value (Fig. 8b), the total likelihood field is applied and is able to completely identify the interference bins and, thus, remove them from the reflectivity map (Figs. 8a and c). The fuzzy logic QC approach is expected to further refine the interferences identification process, but the most important point is the approach is not allowed to eliminate weather echoes. Therefore, a weather case without any interference echo is selected to examine the performance of the fuzzy logic QC algorithm. As shown in Fig. 9, at the eastern side of the radar HINA, a weather system without any interference echo was observed. The total likelihood (Fig. 9b) indicates the weather system is characterized by low likelihood of interference echo except at the periphery of the weather system, where some fragmentary echoes, with likelihood $>0.5$, and 

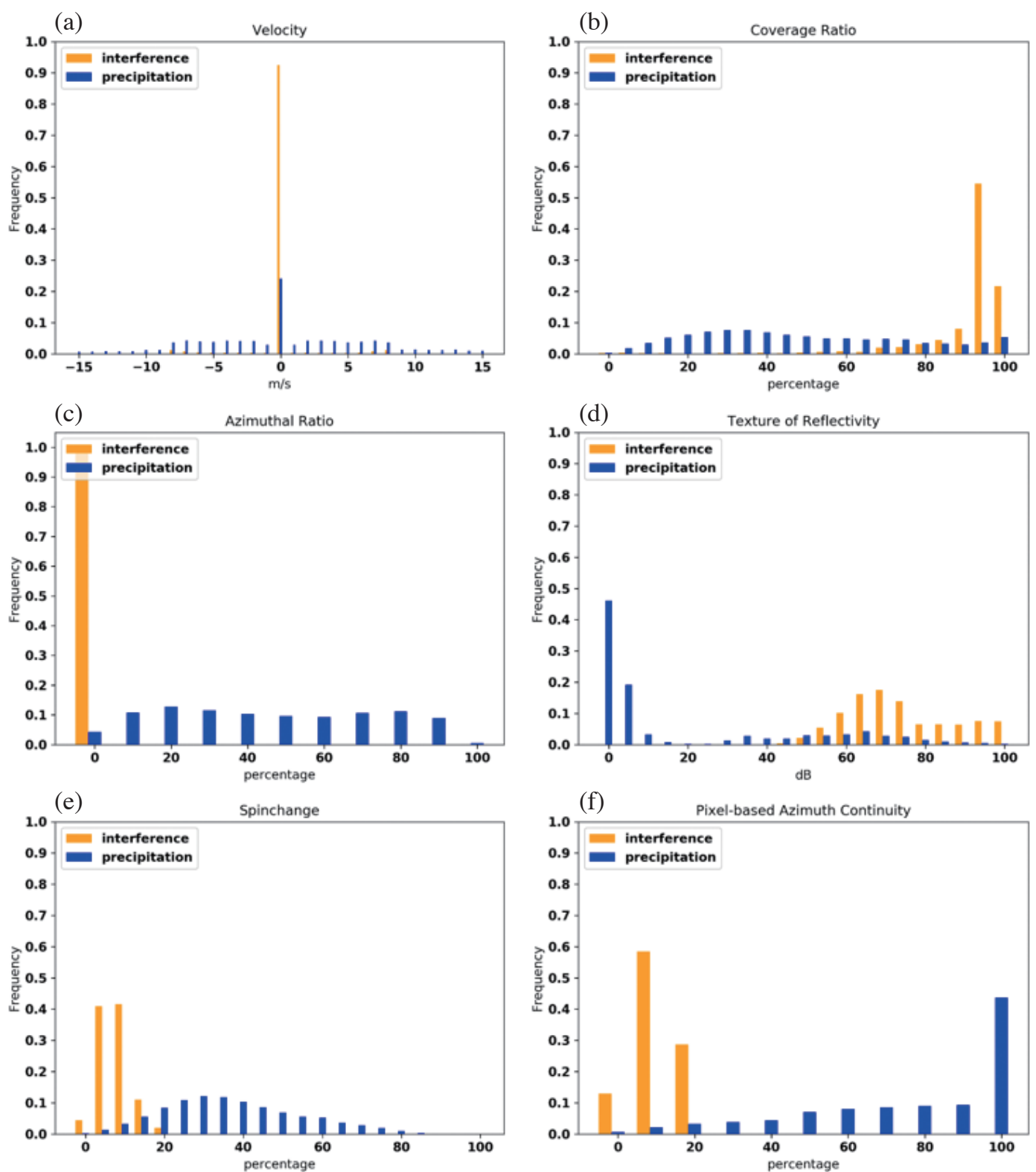

Fig. 4. Frequency distribution functions of 6 features corresponding for the precipitation echoes (blue) and interference echoes (orange) derived from the 500 cases as listed in Table 2. (a) Radial velocity (VEL), (b) Coverage Ratio in Radial (CR), (c) Azimuthal Ratio (AZR), (d) Texture of Reflectivity (RTEX), (e) Spin Change (SC), and (f) Pixel-based Azimuth Continuity (PAC). 

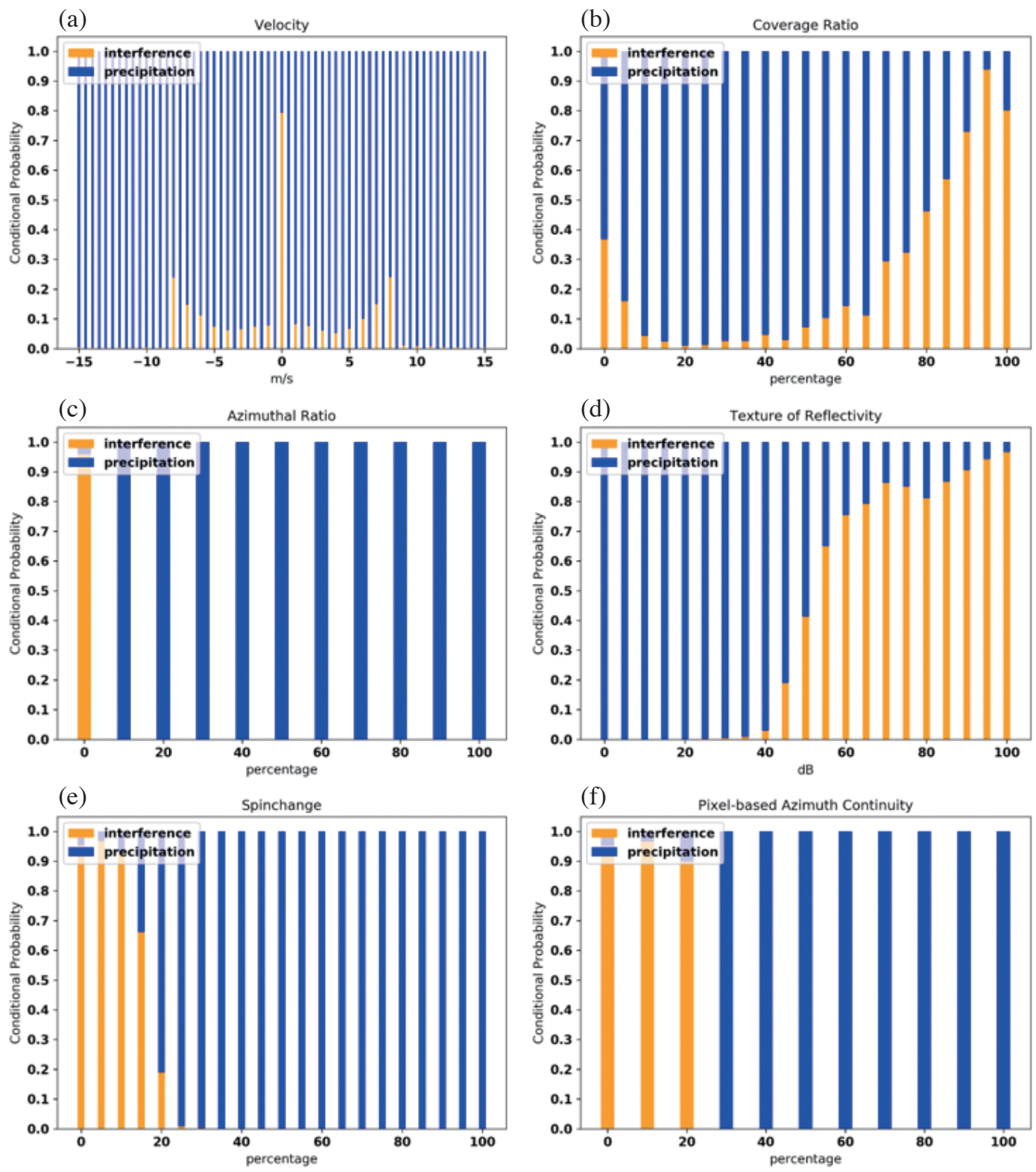

Fig. 5. Conditional probability curves corresponding to precipitation and interference echoes derived from the frequency distribution functions of Fig. 4. 
(a)

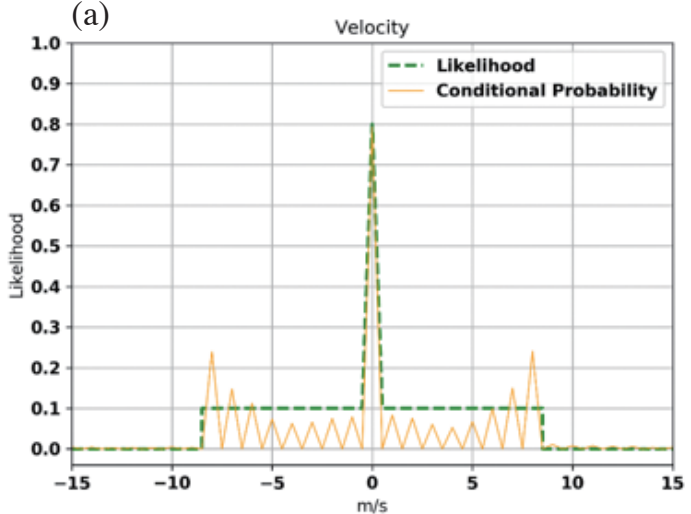

(c)

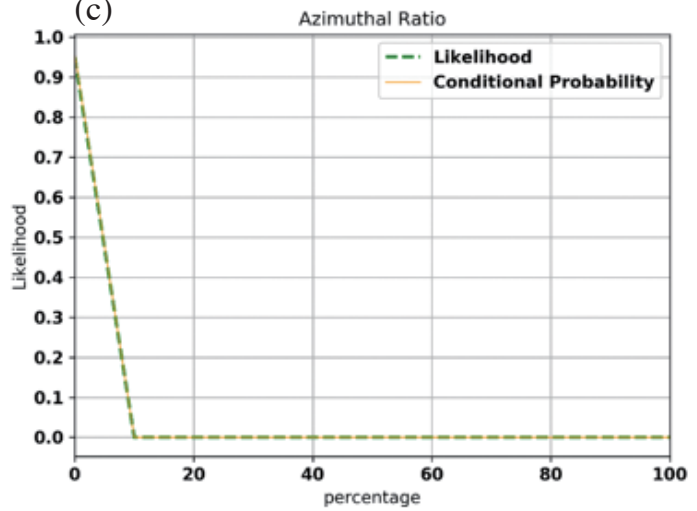

(e) (b)

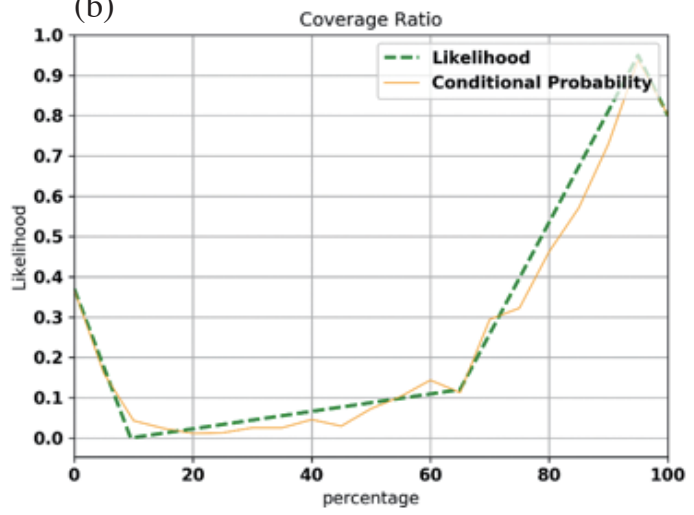

(d)

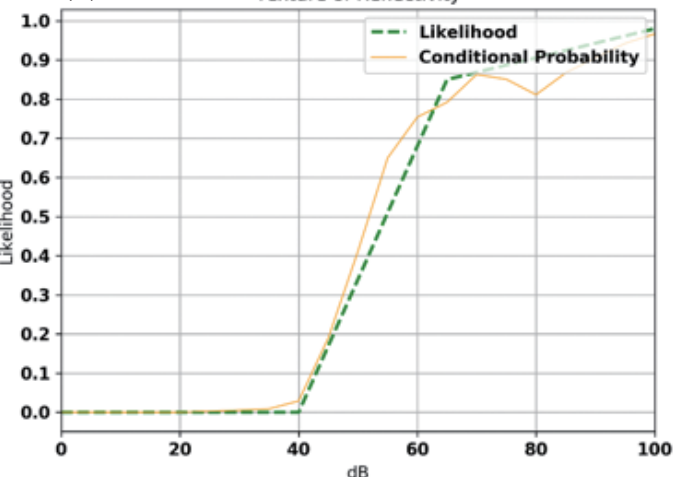

dB

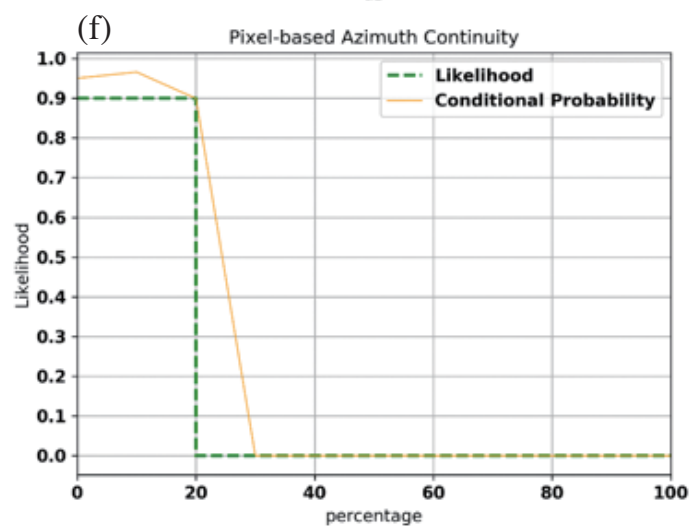

Fig. 6. Fuzzy membership functions corresponding to interference echoes derived from the conditional probability curves of Fig. 5. 


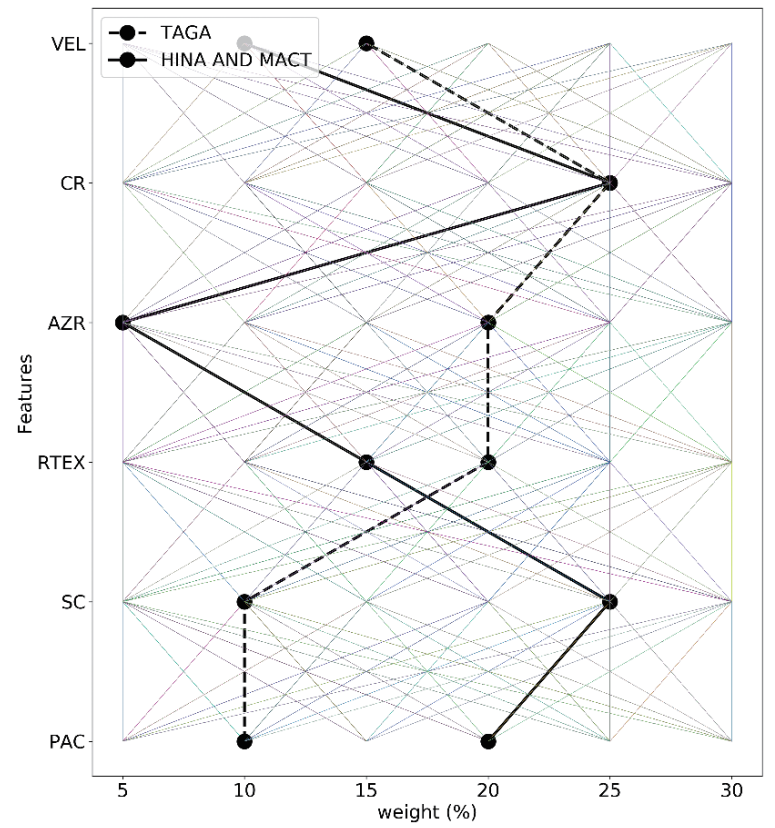

Fig. 7. For the HINA, MACT, and TAGA radars, weights of the 6 features for discriminating interference data bins associated with optimum CSI values were obtained from the fuzzy logic approach. Thin lines indicate all weight combinations. The bold-solid black line are the weights assigned for the 6 features for HINA and MACT radars, and the bold-dotted black line is for TAGA radar.

Table 3. The identifications for hit, miss, and false and the associated error threshold values used in calculating CSI score. NQCed and NunQC are counted by the number of reflectivity $>0 \mathrm{dBZ}$ in a radar file before and after the QC procedures of the fuzzy logic approach. Error threshold used in fuzzy logic algorithm is in bold.

\begin{tabular}{|c|c|c|c|c|c|c|c|}
\hline & \multicolumn{3}{|c|}{ Interference files } & \multicolumn{4}{|c|}{ Weather files } \\
\hline Hit & \multicolumn{3}{|c|}{ NQCed/NunQC $\leq$ Error $_{\text {threshold }}$} & \multicolumn{4}{|c|}{ NQCed/NunQC $\geq 1$-Error ${ }_{\text {threshold }}$} \\
\hline Miss & \multicolumn{3}{|c|}{ NQCed/NunQC > Error threshold } & \multicolumn{4}{|c|}{ none } \\
\hline False & & none & & \multicolumn{4}{|c|}{ NQCed/NunQC $<1-$ Error $_{\text {threshold }}$} \\
\hline & \multicolumn{7}{|c|}{ QC threshold } \\
\hline Error $_{\text {threshold }}(\%)$ & 10 & 5 & 4 & 3 & 2 & 1 & 0 \\
\hline CSI & 0.986 & 0.924 & 0.862 & 0.780 & 0.657 & 0.585 & 0.364 \\
\hline
\end{tabular}
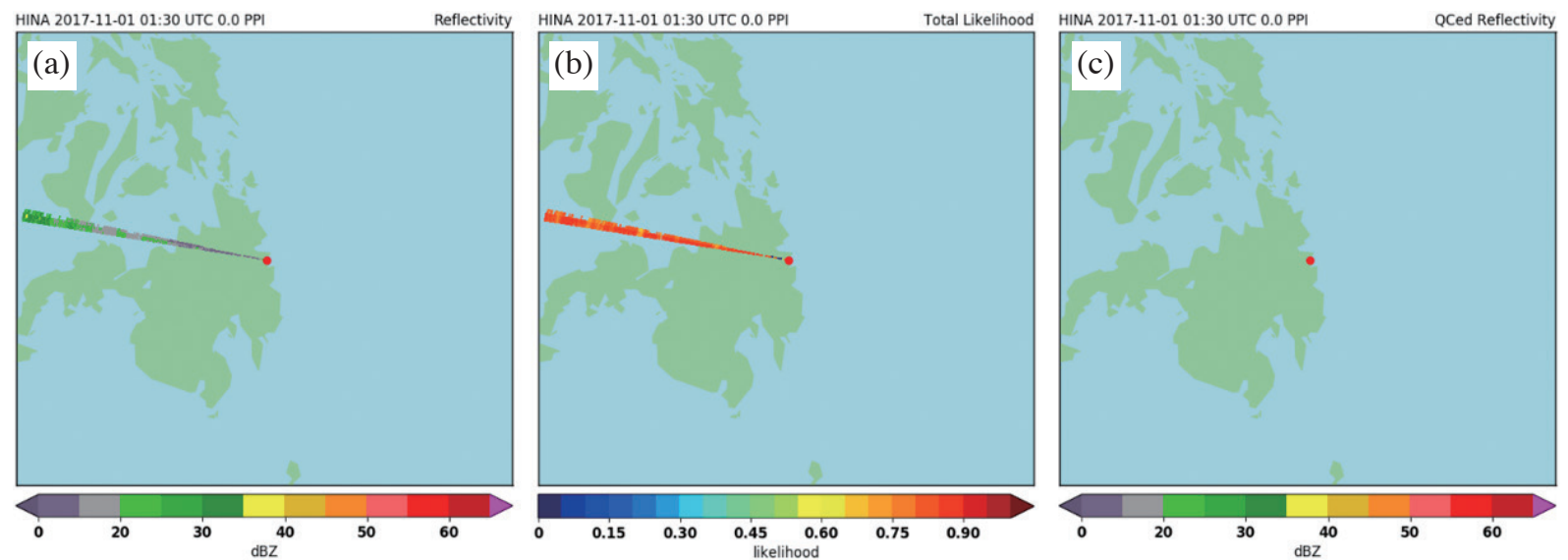

Fig. 8. For the observation from the HINA radar reflectivities at 0 degree elevation at 0130 UTC 01 November 2017, (a) reflectivity field before QCed by fuzzy logic approach; (b) summed likelihood from all 6 membership functions; (c) same as (a) but after QCed. The radar position is indicated by a red dot. 

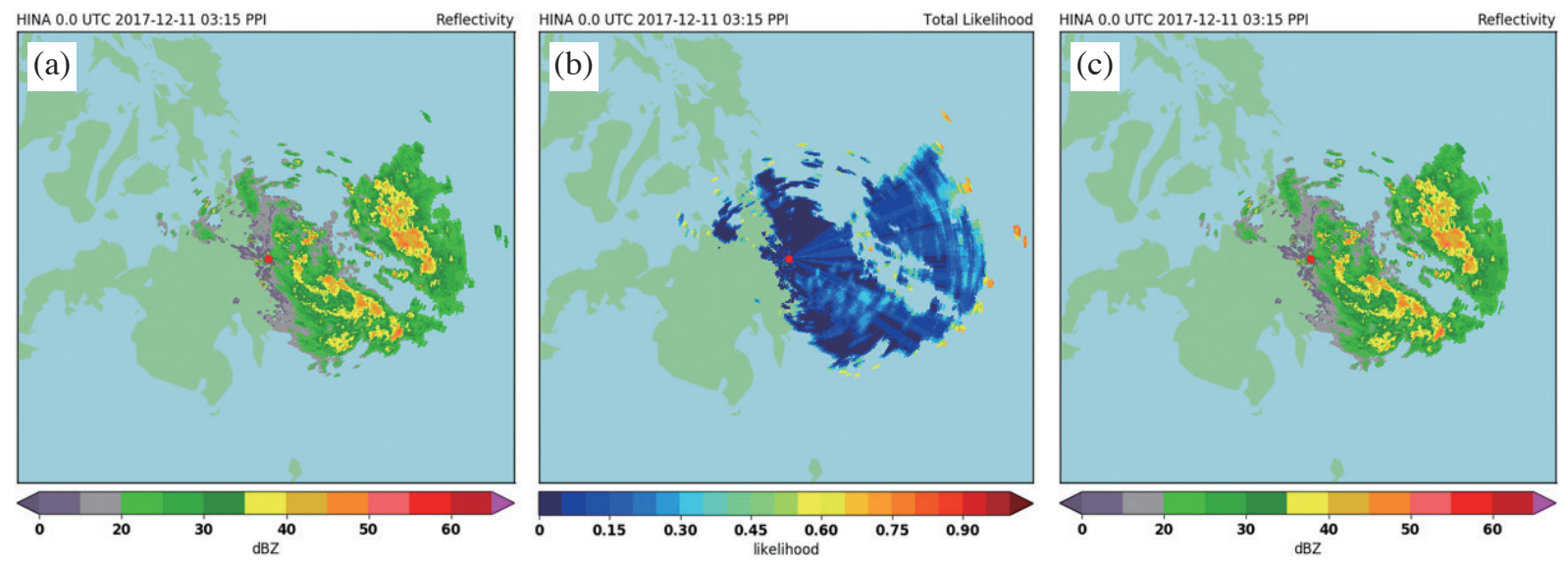

Fig. 9. Same as Fig. 8, but at 0315 UTC 11 December 2017.

are consequently removed (Figs. 9a and c). From these two cases, the total likelihood can be used to circumscribe most of the interference pattern and then remove them from the reflectivity map.

\subsection{Validation Dataset}

In this sub-section, three representative validation cases will be examined here: one is observed by the HINA radar, while the fuzzy logic algorithm also is utilized to remove interference echoes in the other two cases from the MACT and TAGA radars. Incidentally, the fuzzy logic algorithm is developed based on the pure interference and pure weather cases (as listed in Table 2), but the three validation cases are examples of interference embedded in precipitation echoes that are used to evaluate its performance.

The first validation case, observed by the HINA radar, is a line-pattern interference case embedded in weather patches. The respective likelihoods values of the features VEL, RTEX, and PAC (Figs. 10a, d, and f) followed by their membership functions (Figs. 6a, d, and f) as implement the definitions of interference in radar bins are shown. These are three features that contribute to the likelihood value and possess the ability to differentiate interference from precipitating reflectivities. After the fuzzy logic QC procedure, most interferences are removed while precipitation data are kept (Figs. $10 \mathrm{~g}$ and i) as shown by the total likelihood (Fig. 10h), nevertheless some reflectivity spots from interference remain, which can then be filtered via a sequential despeckling procedure.

Another representative interference case is a case with some striking linear interferences that was observed by the MACT radar. These interferences are labeled using features AZR, SC, and PAC (Figs. 11c, e, and f). Compared to the prior case, this scenario produce better likelihood fields to define the interference pattern and remove them within the reflectivity map (see Figs. 11g and i), and, additionally, the weather patches in the east of the MACT radar are kept after the fuzzy logic QC algorithm. Incidentally, identifying interference using the feature values, or likelihood values, is more defined (and hence more likely) if these values exists within a narrow range. For this interference case, the feature value ranges are shown in features AZR, SC, and PAC (Figs. 11c, e, and f) and are narrow. Therefore, after weighting and summing to obtain the final likelihood value (Fig. 11h), the total likelihood is able to be used to identify the interference bins within the reflectivity map (see Figs. 11g and i).

The last interference case explored here, is the typhoon Doksuri (2017) case observed by the TAGA radar with interference lines embedded in precipitation echoes as shown in Fig. 12g. The TAGA radar used a different set of weights (Fig. 7) gotten from 100 weather cases and 100 interference echo cases, due to the weights from HINA employed in TAGA radar having a low CSI rate (not shown). It is speculated that the big altitude difference between HINA and TAGA (Table 1) caused the observational features changed, and thus the low effective score. The calculated likelihood in Figs. 12a to f shows that except the feature PAC (Fig. 12f), other features can be used to identify the interference area, especially features $\mathrm{CR}$ and AZR (Figs. 12b and c) which show separable interference and precipitation value ranges. Features $\mathrm{CR}$ and AZR more accurately assign high likelihoods in interference patterns than that of VEL, RTEX, and SC (Figs. 12a, d, and e), which identify some parts of precipitation data points as interferences. All of the likelihood values are multiplied with each own weight and summed up to produce an overall likelihood value as shown in Fig. 12h. Data bins having total likelihood larger than $50 \%$ are identified as interferencecontaminated gates and were discarded (Figs. 12g and i). Based on these results, the fuzzy logic algorithm QC performs effectively in this complex scenario at keeping the precipitation patches. 
HINA $1^{\text {st }}$ tilt
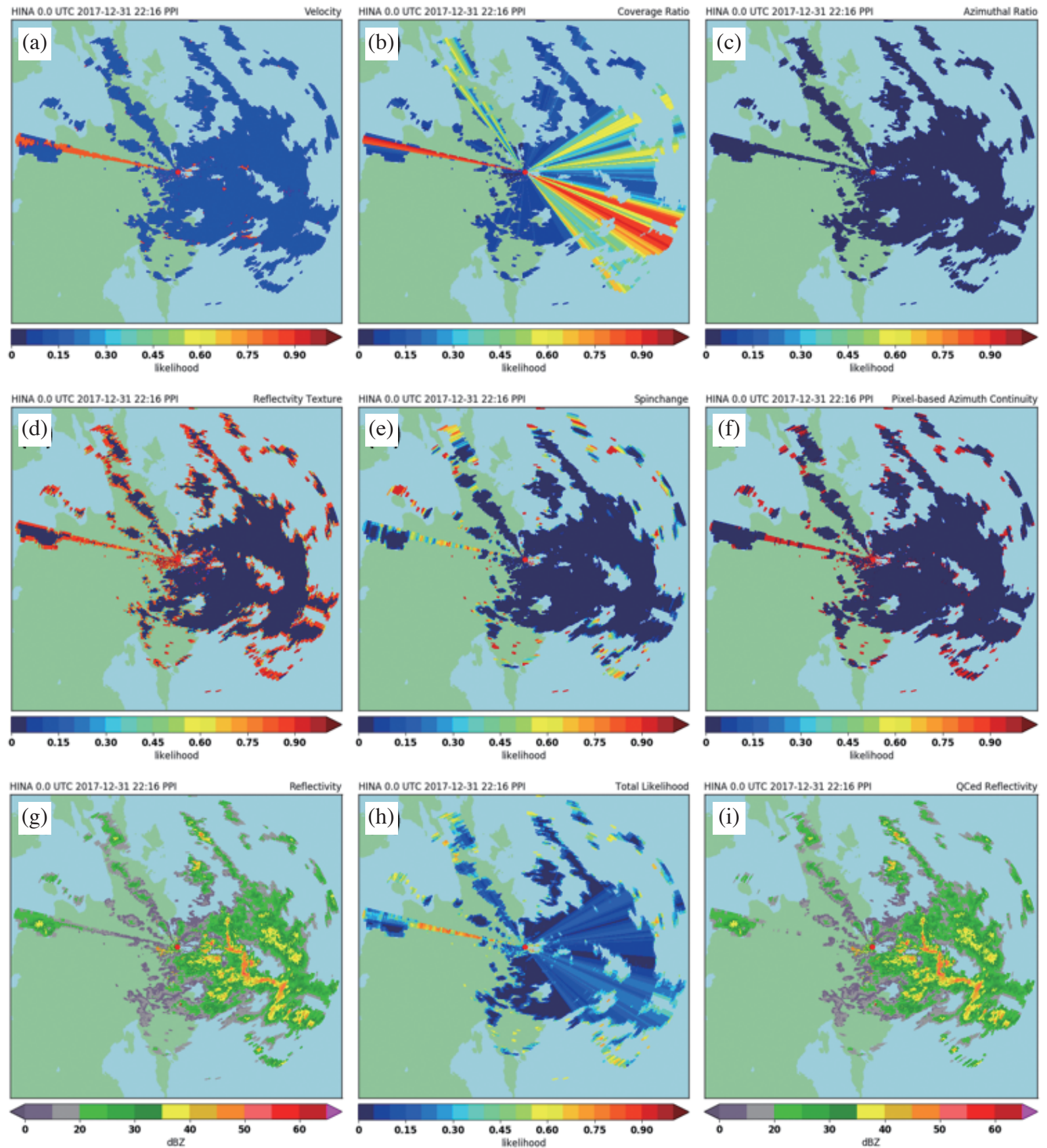

Fig. 10. Observation from the HINA radar at 0 degree elevation at 2216 UTC 31 December 2017. (a) Doppler velocity (VEL), (b) Coverage Ratio in Radial (CR), (c) Azimuthal Ratio (AZR), (d) Texture of Reflectivity (RTEX), (e) Spin Change (SC), and (f) Pixel-based Azimuth Continuity (PAC) likelihood images from the corresponding membership functions; (g) reflectivities before QC by fuzzy logic approach; (h) summed likelihood from all 6 membership functions, (i) same as (g) but after QC. The radar position is indicated by a red dot. 


\section{MACT $1^{\text {st }}$ tilt}
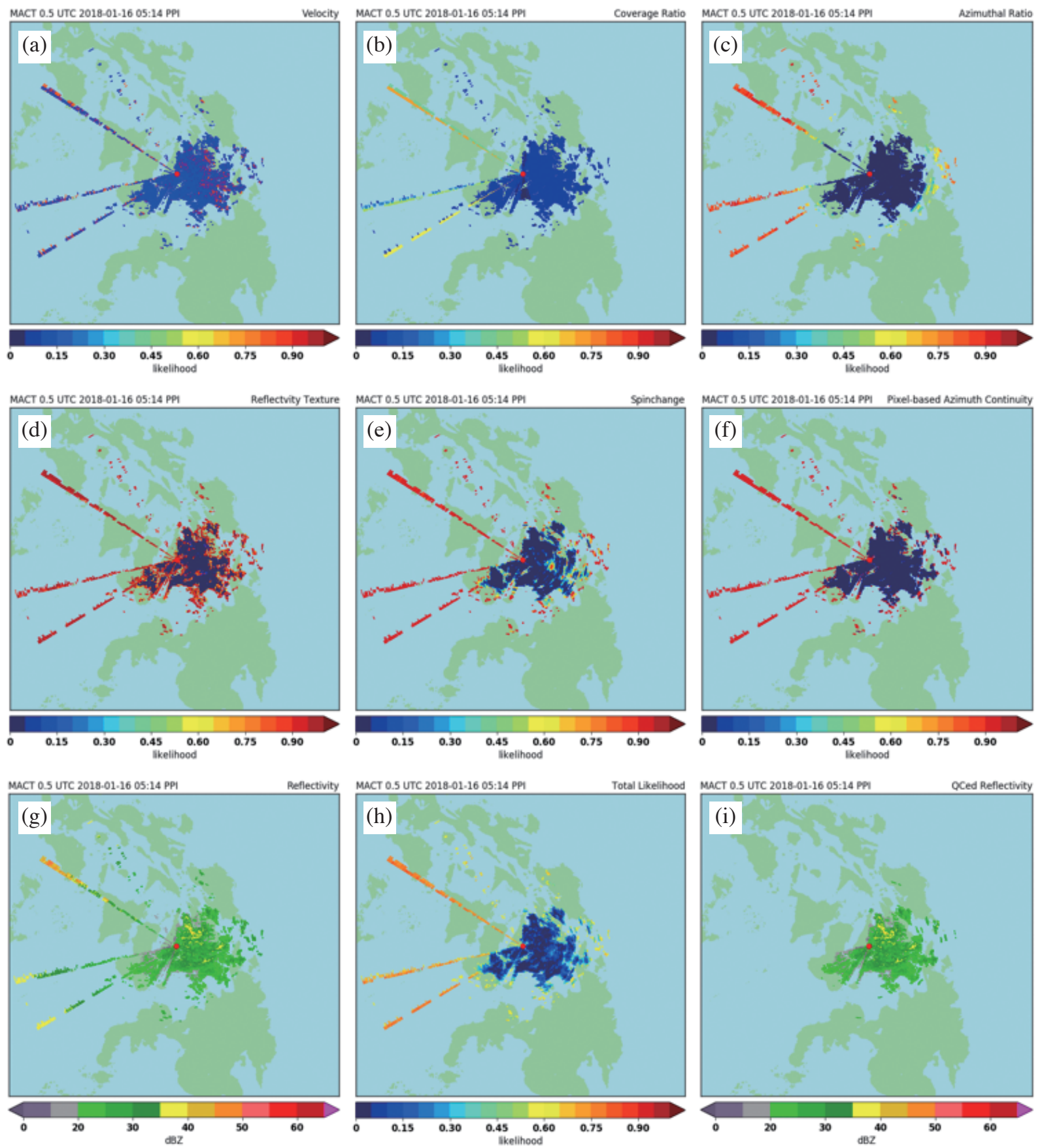

Fig. 11. Same as Fig. 10, but for the MACT radar reflectivities at 0.5 degree elevation at 0514 UTC 16 January 2018. 
TAGA $1^{\text {st }}$ tilt
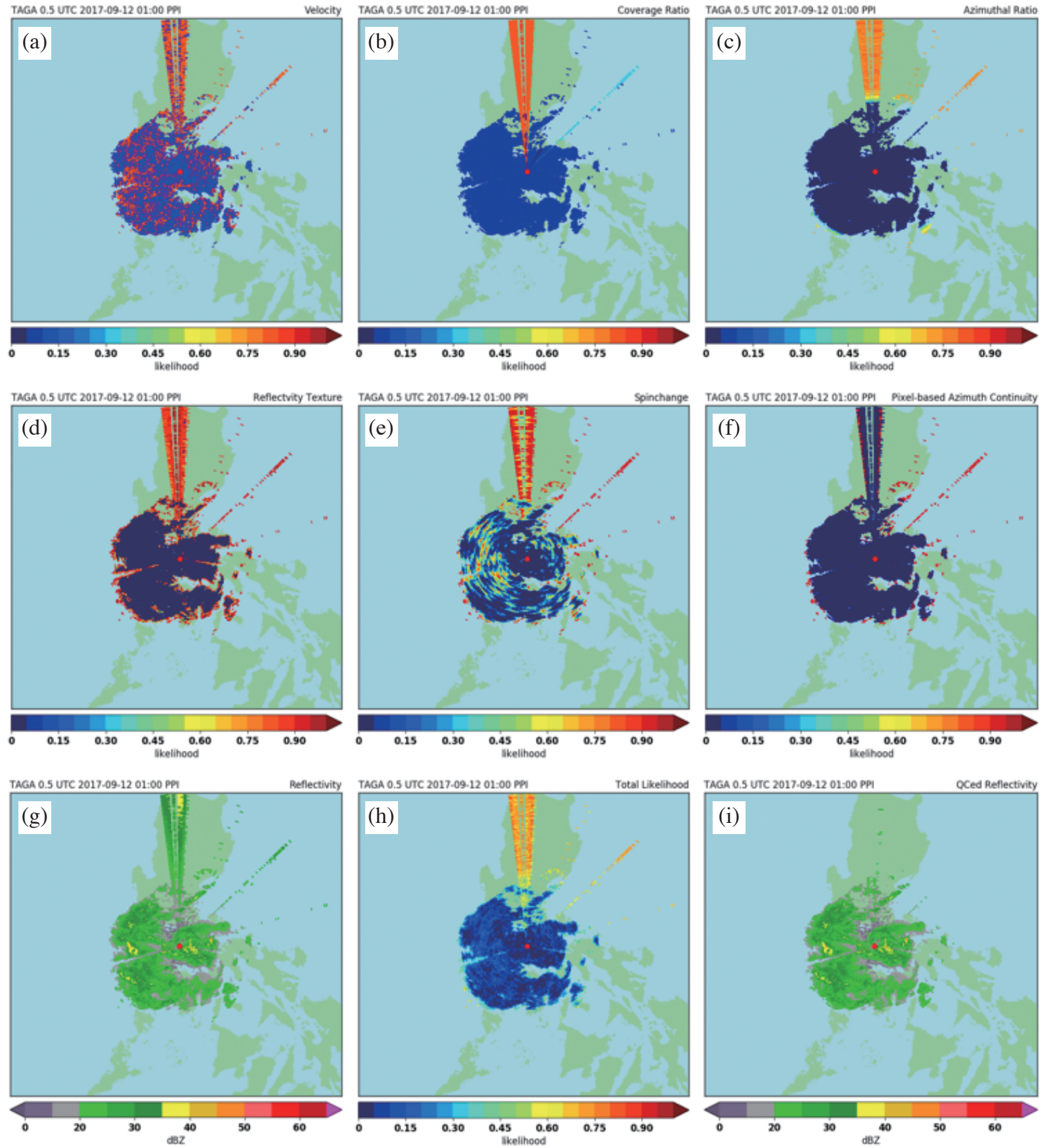

Fig. 12. Same as Fig. 10, but for the TAGA radar reflectivities at 0.5 degree elevation at 0100 UTC 12 September 2017. 


\subsection{Effect on Radar QPE}

Radar precipitation estimates are usually computed from reflectivity observations on the so-called hybrid scans. The hybrid scan of a radar is the lowest radar bins that do not have significant blockages or clutter contaminations (O’Bannon 1997; Maddox et al. 2002; Chang et al. 2009) and also for improving quantitative precipitation estimation in complex orography (Morin and Gabella 2007; Chang et al. 2009). To evaluate the impact of interference echoes, the hybrid scan table is constructed for the radar TAGA as shown in Fig. 13. Figure 13a shows that there are two narrow beam blockage wedges to the east and the south of the radar resulted from topography, the highest tilt is the third elevation, $1.5^{\circ}$. The hybrid scan heights (Fig. 13b) display the nearly concentric circle pattern, which means the blockage effect is insignificant for the TAGA radar. Based on the hybrid scans, the lowest radar bins of the radar data are obtained, and taken into counting the 1 hour accumulated QPE through the $Z-R$ relationship $Z=32.5 R^{1.65}$ (Xin et al. 1997), and presented in Fig. 14. In this instance (Fig. 14a), interference echoes are detected, hence any attempt to estimate accumulate precipitation is severely compromised. Therefore, interference problems such as those in this scenario that are created by one single interference file can suddenly appear in any QPE file and can propagate for an indeterminate time. After the developed fuzzy logic QC procedures, the error caused by the interference echoes can be identified and thus eliminated from ever propagating (Fig. 14b).

Hybrid scan heights from all radars are mosaiced to assess the radar coverage in the Philippines area (Fig. 15). At any given location within the radar map, the lowest hybrid scan height among all radars is chosen. In the Philippines, the hybrid scan height is mostly under $4 \mathrm{~km}$ above ground level (AGL). The result in Fig. 15 suggests that the current weather radar network in Philippines is can be improved for QPE, but it is acceptable under the current circumstances. Figure 16 is the mosaiced 1-hour radar QPE used to evaluate the impact of the fuzzy logic QC procedure. It shows advantages of using fuzzy logic QC algorithm in reducing the impact of interference echoes. It is remarkable that the extremely severe contamination which manifests as QPE with $>200 \mathrm{~mm} \mathrm{hr}^{-1}$ was caused by the non-precipitation echoes (not shown) such as sea clutter or ground clutter. These echoes are perhaps eliminated by selectively adjusting features or weights that suppresses these echo types.

\section{SUMMARY AND CONCLUSION}

Despite elaborate and sophisticated efforts in radar data quality assurance for $\mathrm{AP}$ and $\mathrm{GC}$, the challenges of contamination by interference remain a serious problem, especially for situations where interference echoes are embedded in precipitation echoes and, consequently, precipitation accumulation algorithms and even downstream applications. Therefore, in this study, the membership functions were calculated using comprehensively objective diagnoses approach, e.g., producing conditional probability curves from feature curves of interference echoes. The weights for each membership function to differentiate between interference and precipitation echoes were also objectively determined. To create an operational algorithm for eliminating interference echoes, a fuzzy logic QC approach is advantageous, as the algorithm as simple while the inherent performance is acceptable.

This study focused on removing the root cause issue, the interference, and has presented a thorough analysis of the fuzzy logic QC algorithm for doing so in section 3. In this study, the performance of fuzzy logic QC approach are evaluated based on different radars in the Philippines, and
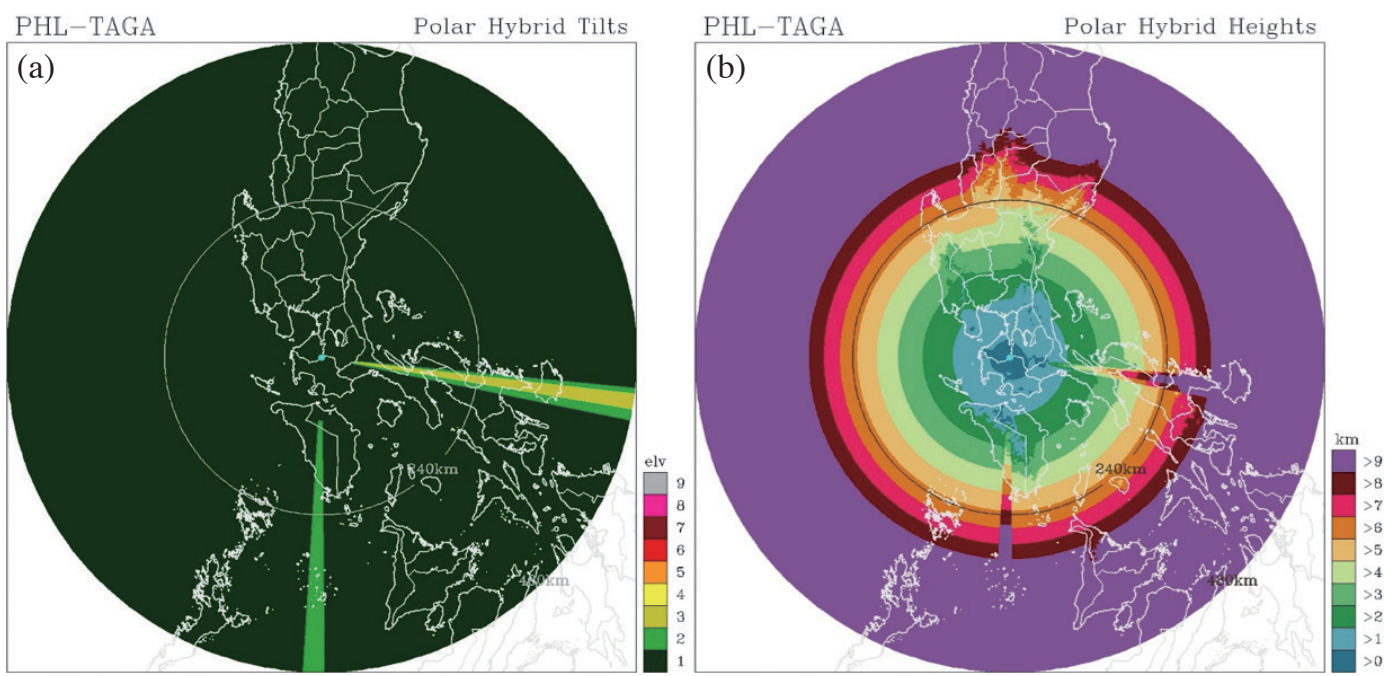

Fig. 13. (a) Hybrid scans and (b) the corresponding hybrid scan heights for the TAGA radar. The radar position is indicated by a cyan dot. 

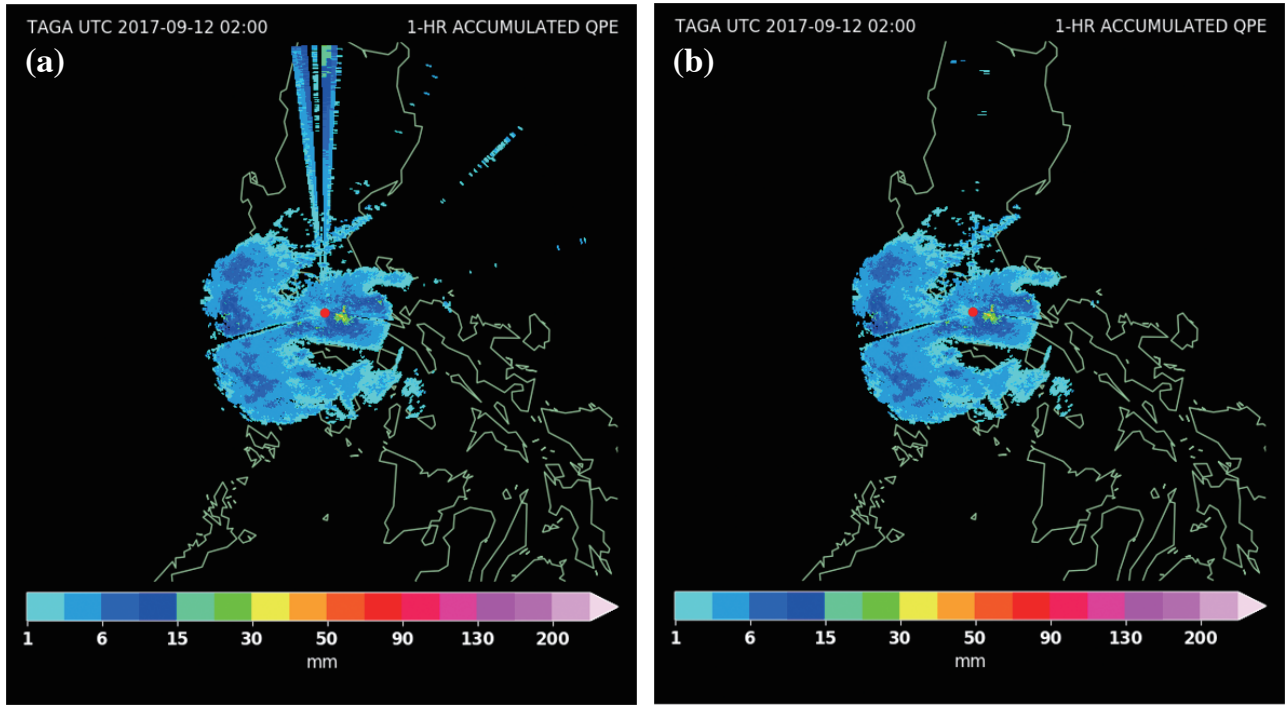

Fig. 14. The radar one hour QPE from the hybrid reflectivities of the TAGA radar at 0200 UTC 12 September 2017, (a) before and (b) after QC by fuzzy logic approach. The radar position is indicated by a red dot.

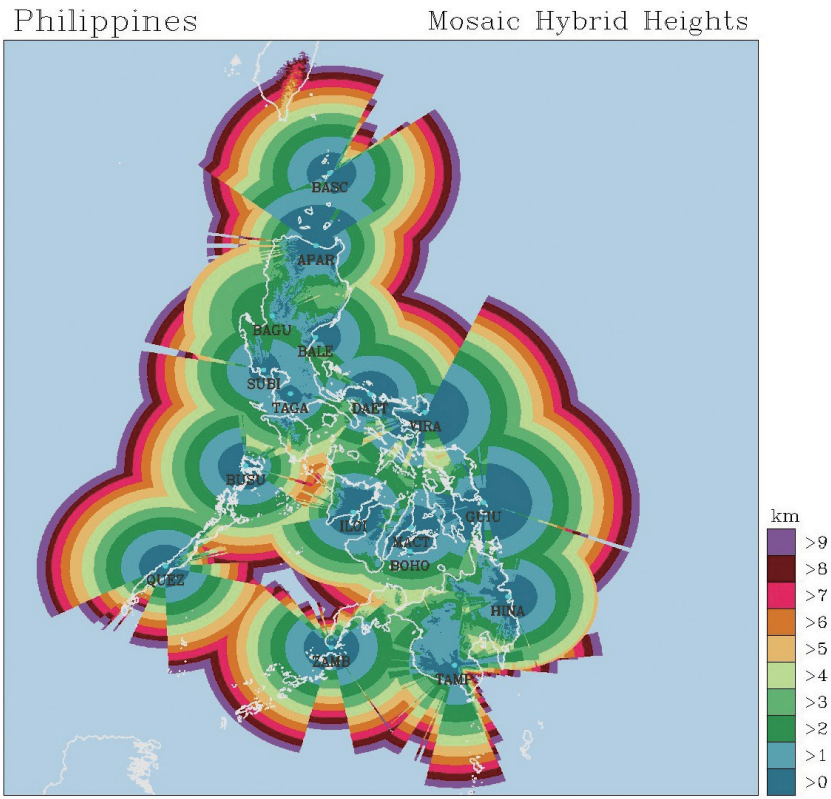

Fig. 15. Mosaiced hybrid scan heights from the 17 radars in Philippines area. Radar sites are marked using cyan dots and labeled with their abbreviated symbol identifier. The coastal area is contoured by white line. 

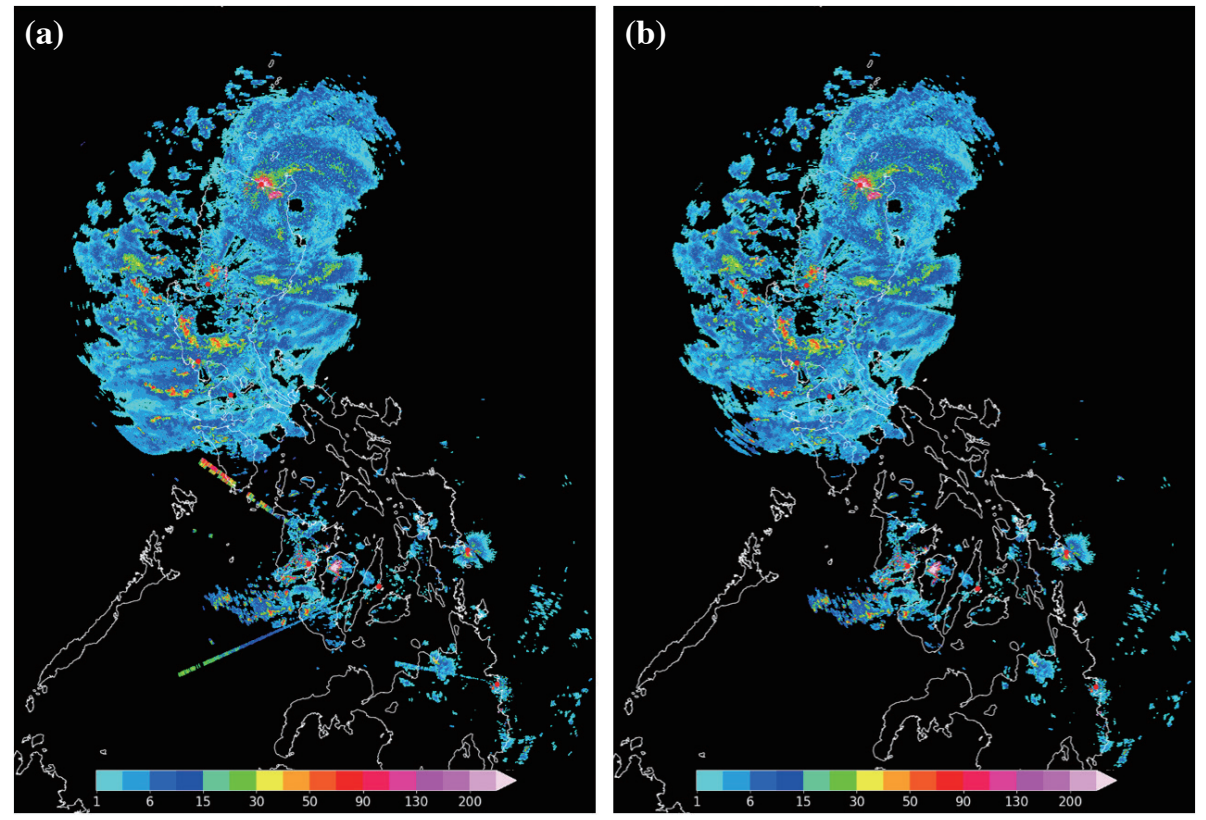

Fig. 16. Same as Fig. 14, but for the mosaic hybrid QPE at 1800 UTC 14 September 2018.

has shown an efficient ability to discriminate interferencecontaminated gates from precipitation patches and then remove them. The algorithm appears to work effectively in situations in both situations where interference echoes are either separated from or embedded within precipitation echoes. It truly improves the radar quantitative precipitation estimate (QPE).

The performance of the fuzzy logic QC showed recognizable improvement in the quality of reflectivity map, while the objective approach helps to systematically decrease the interference targets. Incidentally, a despeckling procedure can be added to remove small or isolated noises that remain. Beyond these refinements, methods for improving the fuzzy logic QC algorithm exists if needed, such as adjustments in feature weights for different radar are perhaps necessary, by including more membership functions that may include their own trained weights and even to other non-precipitation echo contaminated like ground or sea clutter.

Acknowledgements In partnership of Central Weather Bureau (CWB) and PAGASA under VOTE (Volcanos, Ocean, Typhoon, and Earthquake) meteorological program, fuzzy logic approach was developed to remove interference patterns from weather echoes to improve PAGASA weather operations.

\section{REFERENCES}

Berenguer, M., D. Sempere-Torres, C. Corral, and R. Sánchez-Diezma, 2006: A fuzzy logic technique for identifying nonprecipitating echoes in radar scans. $J$. Atmos. Ocean. Technol., 23, 1157-1180, doi: 10.1175/ jtech1914.1. [Link]

Bermowitz, R. J. and E. A. Zurndorfer, 1979: Automated guidance for predicting quantitative precipitation. Mon. Weather Rev., 107, 122-128, doi: 10.1175/1520-0493(1979)107<0122:AGFPQP>2.0. $\mathrm{CO} ; 2$. [Link]

Chang, P.-L., P.-F. Lin, B. J.-D. Jou, and J. Zhang, 2009: An application of reflectivity climatology in constructing radar hybrid scans over complex terrain. J. Atmos. Ocean. Technol., 26, 1315-1327, doi: 10.1175/2009jtecha1 162.1. [Link]

Cho, J. Y. N., 2017: A New Radio Frequency Interference Filter for Weather Radars. J. Atmos. Ocean. Technol., 34, 1393-1406, doi: 10.1175/jtech-d-17-0028.1. [Link]

Cho, Y.-H., G. W. Lee, K.-E. Kim, and I. Zawadzki, 2006: Identification and removal of ground echoes and anomalous propagation using the characteristics of radar echoes. J. Atmos. Ocean. Technol., 23, 1206-1222, doi: 10.1175/jtech1913.1. [Link]

Cornman, L. B., R. K. Goodrich, C. S. Morse, and W. L. Ecklund, 1998: A fuzzy logic method for improved moment estimation from Doppler spectra. J. Atmos. Ocean. Technol., 15, 1287-1305, doi: 10.1175/1520-0426(1998)015<1287:AFLMFI>2.0. $\mathrm{CO} ; 2$. [Link]

Donaldson, R. J., R. M. Dyer, and M. J. Kraus, 1975: An objective evaluator of techniques for predicting severe weather events. $9^{\text {th }}$ Conference Severe Local Storm, 
American Meteorological Society, Norman, OK, 321326. Available at https://ntrl.ntis.gov/NTRL/dashboard/searchResults/titleDetail/ADA015773.xhtml.

Droegemeier, K. K., J. D. Smith, S. Businger, C. Doswell III, J. Doyle, C. Duffy, E. Foufoula-Georgiou, T. Graziano, L.D.James, V.Krajewski, M.LeMone, D.Lettenmaier, C. Mass, R. Pielke Sr., P. Ray, S. Rutledge, J. Schaake, and E. Zipser, 2000: Hydrological aspects of weather prediction and flood warnings: Report of the ninth prospectus development team of the U.S. Weather Research Program. Bull. Amer. Meteorol. Soc., 81, 26652680, doi: 10.1175/1520-0477(2000)081<2665:HAO WPA $>2.3 . C O ; 2$. [Link]

Ellis, S., C. Kessinger, T. D. O'Bannon, and J. Van Andel, 2003: Mitigating ground clutter contamination in the WSR-88D. Preprints CD, 19th International Conference on Interactive Information and Processing Systems (IIPS) for Meteorology, Oceanography, and Hydrology, American Meteorological Society, Long Beach, CA. Available at https://citeseerx.ist.psu.edu/viewdoc/down load?doi=10.1.1.547.5843\&rep=rep1\&type=pdf.

Gourley, J. J., P. Tabary, and J. P. du Chatelet, 2007: A fuzzy logic algorithm for the separation of precipitating from nonprecipitating echoes using polarimetric radar observations. J. Atmos. Ocean. Technol., 24, 1439-1451, doi: 10.1175/jtech2035.1. [Link]

Grecu, M. and W. F. Krajewski, 2000: An efficient methodology for detection of anomalous propagation echoes in radar reflectivity data using neural networks. J. Atmos. Ocean. Technol., 17, 121-129, doi: 10.1175/1520-0426(2000)017<0121:AEMFDO>2.0. $\mathrm{CO} ; 2$. [Link]

Heske, T. and J. Heske, 1996: Fuzzy Logic for Real World Design, $1^{\text {st }}$ Edition, Annabooks, 428 pp.

Kessinger, C., S. Ellis, and J. Van Andel, 2001: NEXRAD Data Quality: The AP clutter mitigation scheme. 30th International Conference on Radar Meteorology, American Meteorological Society, Munich, Germany, 707-709. Available at https://ams.confex.com/ ams/30radar/techprogram/paper 22012.htm.

Kessinger, C., S. Ellis, and J. Van Andel, 2002: NEXRAD Data Quality: An update on the AP clutter mitigation scheme. 18th International Conference on Interactive Information and Processing Systems (IIPS) for Meteorology, Oceanography, and Hydrology, American Meteorological Society, Orlando, 127-129. Available at https://www.academia.edu/27624847.

Kessinger, C., S. Ellis, and J. Van Andel, 2003: The radar echo classifier: A fuzzy logic algorithm for the WSR88D. 3rd Conference on Artificial Intelligence Applications to the Environmental Science, American Meteorological Society, Long Beach, CA. Available at https://ams.confex.com/ams/annual2003/techprogram/ paper $54946 . \mathrm{htm}$.

Kilambi, A., F. Fabry, and V. Meunier, 2018: A simple and effective method for separating meteorological from nonmeteorological targets using dual-polarization data. J. Atmos. Ocean. Technol., 35, 1415-1424, doi: 10.1175/jtech-d-17-0175.1. [Link]

Lakshmanan, V., 2000: Using a genetic algorithm to tune a bounded weak echo region detection algorithm. $J$. Appl. Meteorol., 39, 222-230. Available at https:// journals.ametsoc.org/view/journals/apme/39/2/1520045020000390222 uagatt 2.0.co 2.xml.

Lakshmanan, V., A. Fritz, T. Smith, K. Hondl, and G. Stumpf, 2007: An automated technique to quality control radar reflectivity data. J. Appl. Meteorol. Climatol., 46, 288-305, doi: 10.1175/jam2460.1. [Link]

Lee, G., Y.-H. Cho, K.-E. Kim, and I. Zawadzki, 2005: Identification and removal of non-precipitation echoes using the characteristics of radar echoes. 32nd Conference on Radar Meteorology, American Meteorological Society, Albuquerque, NM. Available at https:// www.researchgate.net/publication/228401246 4R 3 IDENTIFICATION AND REMOVAL OF NONPRECIPITATION ECHOES USING THE CHARACTERISTICS OF RADAR ECHOES.

Lee, R., G. D. Bruna, and J. Joss, 1995: Intensity of ground clutter and of echoes of anomalous propagation and its elimination. 27th Conference on Radar Meteorology, American Meteorological Society, Vail, CO , 651-652.

Lin, P.-F., P.-L. Chang, B. J.-D. Jou, J. W. Wilson, and R. D. Roberts, 2012: Objective prediction of warm season afternoon thunderstorms in northern Taiwan using a fuzzy logic approach. Weather Forecast., 27, 11781197, doi: 10.1175/waf-d-11-00105.1. [Link]

Liu, H. and V. Chandrasekar, 2000: Classification of hydrometeors based on polarimetric radar measurements: Development of fuzzy logic and neuro-fuzzy systems, and in situ verification. J. Atmos. Ocean. Technol., 17, 140-164,doi:10.1175/1520-0426(2000)017<0140:CO$\mathrm{HBOP}>2$.0.CO;2. [Link]

Maddox, R. A., J. Zhang, J. J. Gourley, and K. W. Howard, 2002: Weather radar coverage over the contiguous United States. Weather Forecast., 17, 927-934, doi: 10.1175/1520-0434(2002)017<0927:WRCOTC >2.0. CO;2. [Link]

Morin, E. and M. Gabella, 2007: Radar-based quantitative precipitation estimation over Mediterranean and dry climate regimes. J. Geophys. Res., 112, D20108, doi: 10.1029/2006jd008206. [Link]

O'Bannon, T, 1997: Using a 'terrain-based' hybrid Scan to improve WSR-88D precipitation estimates. $28^{\text {th }}$ Conference on Radar Meteorology, American Meteorological Society, Austin, 506-507.

Ogden, F. L., H. O. Sharif, S. U. S. Senarath, J. A. Smith, 
M. L. Baeck, and J. R. Richardson, 2000: Hydrologic analysis of the Fort Collins, Colorado, flash flood of 1997. J. Hydrol., 228, 82-100, doi: 10.1016/s00221694(00)00146-3. [Link]

Overeem, A., R. Uijlenhoet, and H. Leijnse, 2020: Full-year evaluation of Nonmeteorological echo removal with dual-polarization fuzzy logic for two C-Band radars in a Temperate Climate. J. Atmos. Ocean. Technol., 37, 1643-1660, doi: 10.1175/jtech-d-19-0149.1. [Link]

Panofsky, H. A. and G. W. Brier, 1965: Some Applications of Statistics to Meteorology, The Pennsylvania State University, University Park, PA, 224 pp.

Radhakrishna, B., F. Fabry, and A. Kilambi, 2019: Fuzzy logic algorithms to identify birds, precipitation, and ground clutter in S-band radar data using polarimetric and nonpolarimetric variables. J. Atmos. Ocean. Technol., 36, 2401-2414, doi: 10.1175/jtech-d-19-0088.1. [Link]

Saltikoff, E., J. Y. N. Cho, P. Tristant, A. Huuskonen, L. Allmon, R. Cook, E. Becker, and P. Joe, 2016: The threat to weather radars by wireless technology. Bull. Amer. Meteorol. Soc., 97, 1159-1167, doi: 10.1175/ BAMS-D-15-00048.1. [Link]
Smith, J. A., M. L. Baeck, M. Steiner, B. Bauer-Messmer, W. Zhao, and A. Tapia, 1996: Hydrometeorological assessments of the NEXRAD rainfall algorithms. NOAA National Weather Service Final Report, 55 pp.

Steiner, M. and J. A. Smith, 2002: Use of three-dimensional reflectivity structure for automated detection and removal of nonprecipitating echoes in radar data. J. Atmos. Ocean. Technol., 19, 673-686, doi: 10.1175/1520-0426(2002)019<0673:UOTDRS>2.0. $\mathrm{CO} ; 2$. [Link]

Trapp, R. J. and C. A. Doswell III, 2000: Radar data objective analysis. J. Atmos. Ocean. Technol., 17, 105-120, doi: 10.1175/1520-0426(2000)017<0105:RDOA>2.0. $\mathrm{CO} ; 2$. [Link]

Xin, L., G. Reuter, and B. Larochelle, 1997: Reflectivityrain rate relationships for convective rainshowers in Edmonton: Research note. Atmos.-Ocean, 35, 513521, doi: 10.1080/07055900.1997.9649602. [Link]

Ye, B.-Y., G. Lee, and H.-M. Park, 2015: Identification and removal of non-meteorological echoes in dual-polarization radar data based on a fuzzy logic algorithm. Adv. Atmos. Sci., 32, 1217-1230, doi: 10.1007/s00376015-4092-0. [Link] 